\title{
Uncertainty analysis of the CPA and a quadrupolar CPA equation of state - With emphasis on $\mathrm{CO} 2$
}

Bjørner, Martin G.; Sin, Gürkan; Kontogeorgis, Georgios M.

Published in:

Fluid Phase Equilibria

Link to article, DOI:

10.1016/j.fluid.2015.12.037

Publication date:

2016

Document Version

Peer reviewed version

Link back to DTU Orbit

Citation (APA):

Bjørner, M. G., Sin, G., \& Kontogeorgis, G. M. (2016). Uncertainty analysis of the CPA and a quadrupolar CPA equation of state - With emphasis on CO2. Fluid Phase Equilibria, 414, 29-47.

https://doi.org/10.1016/j.fluid.2015.12.037

\section{General rights}

Copyright and moral rights for the publications made accessible in the public portal are retained by the authors and/or other copyright owners and it is a condition of accessing publications that users recognise and abide by the legal requirements associated with these rights.

- Users may download and print one copy of any publication from the public portal for the purpose of private study or research.

- You may not further distribute the material or use it for any profit-making activity or commercial gain

- You may freely distribute the URL identifying the publication in the public portal 


\title{
Uncertainty analysis of the CPA and a quadrupolar $\mathrm{CPA}$ equation of state - with emphasis on $\mathrm{CO}_{2}$
}

\author{
Martin G. Bjørner ${ }^{a, *}$, Gürkan Sin ${ }^{\mathrm{b}}$, Georgios M. Kontogeorgis ${ }^{\mathrm{a}}$ \\ ${ }^{a}$ Center for Energy Resources Engineering (CERE), Department of Chemical and \\ Biochemical Engineering, Technical University of Denmark, Building 229, DK-2800 Kgs. \\ Lyngby, Denmark. \\ ${ }^{b}$ CAPEC-PROCES Research Center, Department of Chemical and Biochemical \\ Engineering, Technical University of Denmark, Building 227, DK-2800 Kgs. Lyngby, \\ Denmark.
}

\begin{abstract}
The parameters of thermodynamic models, such as the cubic plus association (CPA) equation of state, are subject to uncertainties due to measurement errors in the experimental data that the models are correlated to. More importantly as the number of adjustable parameters increase, the parameter estimation problem becomes more complicated due to parameter identifiability issues. In this work the uncertainties in the pure compound parameters of $\mathrm{CO}_{2}$ are investigated using several different CPA approaches, including a new quadrupolar CPA. The uncertainties are estimated using both least squares estimation and the bootstrap method for parameter estimation. The uncertainties in the parameters estimated from the bootstrap method are propagated to physical property and vapor liquid equilibrium predictions using Monte Carlo simulations.

The results indicate that both the pure compound parameter uncertainty and the propagated uncertainty is negligible for the modeling approaches which employ three adjustable parameters. For modeling approaches with more than three adjustable parameters, however, there may be significant uncertainties in the pure compound parameters, as well as a high degree of correlation between the adjustable parameters. This results in significant propagated errors
\end{abstract}

\footnotetext{
* Corresponding author

Email address: mgabj@kt.dtu.dk (Martin G. Bjørner)
}

Preprint submitted to Fluid Phase Equilibria

December 31, 2015 
for certain output properties. To reduce the uncertainty in the adjustable model parameters the heat of vaporization was included as additional correlation data. This resulted in parameter distributions which followed a normal distribution more closely, however, the correlation between the adjustable parameters remained high. Overall the results indicate, that it is important to report parameter uncertainties together with their correlation matrix when a model is developed, so that better informed decisions can be made, for instance about which model extension, or association scheme should be employed.

Keywords: equation of state, $\mathrm{CPA}$, quadrupole, $\mathrm{CO}_{2}$, Parameter Estimation, Propagation of uncertainty

\section{Introduction}

The pure compound parameters of advanced thermodynamic models such as the cubic plus association (CPA) equation of state (EoS) [1] and the Statistical Association Fluid Theory (SAFT) [2] are typically correlated to the saturated 5 vapor pressure and saturated liquid densities using a least squares (LSQ) minimization approach in which, presumably, unique parameters are estimated. Even the most accurate experiments, however, are subject to measurement errors. Moreover the parameters may be correlated, so that a change in one parameter can be compensated by a change in another. Consequently the pure compound parameters will be associated with some degree of uncertainty, which is typically assumed insignificant in the majority of studies thus far. Even small errors in the parameters, however, may significantly affect the result of a simulation $[3,4]$. While several researchers have drawn attention to this problem [4-10] surprisingly little work has been done on analysing and quantifying the

15 uncertainty of parameters in thermodynamic models and their effect on physical property and equilibrium calculations.

The work of Whiting and co-workers $[3,11-16]$ is perhaps one of the most notable contributions to uncertainty estimates of thermodynamic models. Using a Monte Carlo approach the authors analysed the effect of uncertainties in ther- 
modynamic data and their effect on process design. More recently Mathias and co-workers [17-19] also investigated the importance of uncertainty propagation for processes such as $\mathrm{CO}_{2}$ capture. Hajipour and co-workers [20-22] estimated the critical properties for a large number of hydrocarbons. The authors took both the experimental uncertainty in the data as well as the correlation between

25 thermodynamic model parameters into account. Subsequently the uncertainties of the binary interaction parameters for 87 binary mixtures were estimated by use of the pure compound uncertainties.

Most research has focused on the propagated error from a thermodynamic model to various unit operations such as distillation columns (e.g. [5, 6, 17, 23]). Uncertainty analysis, however, can also be used for model development and comparison; by comparing the uncertainties of selected physical properties and equilibria for different models, we can compare the models more objectively. For instance when models such as CPA and SAFT are compared, they often perform almost identically and what differences are present may, in many cases, be due 35 to statistical uncertainties in the pure compound parameters of the models, rather than due to one model being superior to the other.

In an effort to improve the performance of models such as SAFT and to obtain a physically more correct model, additional terms are often added to the base EoS. The addition of an extra term typically leads to an increase in 40 the number of adjustable parameters. This may make it difficult to estimate unique pure compound parameters due to high correlations between parameters as well as the possible presence of multiple local minima. One reason for this is that the data used for parameter estimation is too limited in relation to the model complexity, which must be able to predict a wide range of properties 45 besides those its parameters are fitted to. This may be particularly relevant for a molecule such as $\mathrm{CO}_{2}$, for which the vapor pressure curve is very short. Both SAFT and CPA can for instance correlate the saturated vapor pressure and liquid density of $\mathrm{CO}_{2}$ almost within experimental error, using only the three pure compound parameters commonly employed for non-associating molecules.

Unfortunately neither EoS can predict (i.e. $k_{i j}=0$ ) the unusual phase be- 
havior of mixtures containing $\mathrm{CO}_{2}$ very well. The reason for this is believed to be, that $\mathrm{CO}_{2}$ has a large quadrupole moment, which the traditional approaches do not account for. The large quadrupole moment is, for instance, believed to be the reason for the liquid-liquid equilibrium between $\mathrm{CO}_{2}$ and heavy hydrocarbons. During the past decade several quadrupolar terms have been added to the SAFT framework [24-27]. Unfortunately the new terms are often parametrized by using an additional pure compound parameter (such as an 'effective' quadrupolar moment). Other more pragmatic approaches tend to treat $\mathrm{CO}_{2}$ as a self-associating or solvating molecule. Tsivintzelis et al. [28], for instance, demonstrated that such an approach often works quite well, at the cost of two additional pure compound parameters. If the uncertainties in parameters are significant, however, it may be difficult to compare the performance of various modeling approaches, as their differences may be due to the parametrization, rather than the superiority of one model over the other.

65

In this work, the uncertainties in the pure compound parameters of $\mathrm{CO}_{2}$ are systematically evaluated, when different modeling approaches are employed with CPA as the base model. The uncertainty estimates are obtained from either a linear approximation of the covariance matrix of estimated parameters from nonlinear regression (LSQ) or using the Bootstrap method [29]. A Monte Carlo

70 procedure (with latin hypercube sampling (LHS) and Iman-Conover correlation control) is employed to quantify the effect of parameter uncertainty by propagating the uncertainties to various derivative properties and binary equilibrium calculations.

\section{Methods}

75 2.1. Parameter estimation - uncertainty and correlation

The pure compound parameters in CPA are typically fitted to saturated pressures and saturated liquid densities using a weighted LSQ objective function. Ideally experimental data should be used for such correlations, however, more often than not, pure compound correlations, such as that of Span and Wag- 
ner [30], as implemented in the Reference Fluid Thermodynamic and Transport Properties (REFPROP) program [31] and as disseminated through the National Institute of Standards and Technology (NIST) Chemistry Webbook [32] are employed as pseudo-experimental data, since their correlations are accurate to within experimental error for many compounds. While such pseudoexperimental data are a convenient way of quickly obtaining quite accurate data for many compounds, the measurement errors present in the experimental data is lost. Moreover as the number of model parameters is increased and the closeness of fit improves, there is a clear risk of over-fitting.

In this work we investigate and compare the uncertainty when $\mathrm{CO}_{2}$ is treated 90 as either an inert (non-associating, non-quadrupolar) molecule, an associating molecule, and as a quadrupolar molecule. In the first two cases regular CPA is employed with and without association (with focus primarily on scheme $4 \mathrm{C}$ ). In the latter case we employ the quadrupolar $\mathrm{CPA}(\mathrm{qCPA})$ which is a recent extension of CPA to quadrupolar fluids suggested by Bjørner and Kontogeorgis

95 [33]. Equations for CPA and qCPA can be found in appendix Appendix A or in the literature $[1,33-36]$. Two cases are evaluated when $\mathrm{CO}_{2}$ is considered to be a quadrupolar molecule, one where no additional adjustable parameter is employed and one where an additional volumetric parameter is employed. Table 1 summarizes the various approaches and the adjustable parameters involved in each approach.

Table 1: Modeling approaches with CPA, including the number of adjustable pure compound parameters, investigated for $\mathrm{CO}_{2}$.

\begin{tabular}{ccccc}
\hline Designation & Modeling approach & sites on each molecule & no. Adj & Adjustable parameters \\
\hline A & inert & no sites & 3 & $\Gamma^{a}, b_{0}, c_{1}$ \\
$\mathrm{~B}$ & $2 \mathrm{~B}^{b}$ & one positive, one negative & 5 & $\Gamma, b_{0}, c_{1}, \epsilon, \beta$ \\
$\mathrm{C}$ & $3 \mathrm{~B}$ & one positive, two negative & 5 & $\Gamma, b_{0}, c_{1}, \epsilon, \beta$ \\
$\mathrm{D}$ & $4 \mathrm{C}$ & two postive, two negative & 5 & $\Gamma, b_{0}, c_{1}, \epsilon, \beta$ \\
$\mathrm{E}$ & Quadrupolar & point quadrupole & 3 & $\Gamma, b_{0}, c_{1}$ \\
$\mathrm{~F}$ & Quadrupolar & point quadrupole & 4 & $\Gamma, b_{0}, c_{1}, b_{0}^{Q}$ \\
\hline
\end{tabular}

${ }^{a} \Gamma=a_{0} / R b_{0}$

${ }^{b}$ Terminology from Huang and Radosz [37] . 


\subsubsection{The least squares method}

The LSQ method is a frequentist approach, in which the underlying model parameters are assumed to have true fixed values. However, since experimental data are subject to measurement errors these values can only be estimated by probability distributions of the measurement errors with the aid of statistical estimators $[38,39]$. That is, the model parameters are not random but the estimators are, since they depend on the measurements. If is is assumed that the experimental error can be described by a normal distribution with mean equal to the experimental measurement, then the LSQ method is equivalent to minimizing the weighted sum of squares of the difference between measurements (exp) and mathematical model (m):

$$
\min \chi^{2}(\theta)=\sum_{i=1}^{N}\left(\frac{y_{i}^{e x p}-y_{i}^{m}\left(\theta ; T_{i}\right)}{\sigma_{i}^{e x p}}\right)^{2}
$$

where $N$ is the number of experiments, $y_{i}^{e x p}$ is the $i$ th experimental value of an output property, such as the saturated density or saturated pressure, $y_{i}^{m}\left(\theta ; T_{i}\right)$ represent the results from the model at temperature, $T_{i}$, where $\theta$ is a vector of adjustable parameters which depends on the modeling approach. The weight of the $i$ th term is given as the inverse variance of the $i$ th measurement, $\left(\sigma_{e x p, i}^{2}\right)$. In principle $\sigma$ is the total standard deviation including both the uncertainty in dependent and independent variables. In this work, however, we assume that the uncertainty in the independent variables is insignificant, which greatly simplifies the data fitting problem.

Under a linear approximation the covariance matrix of parameter estimators can be calculated from $[40,41]$

$$
\operatorname{Cov}(\hat{\theta}) \approx \frac{\chi^{2}}{N-p}\left(\left(\frac{\partial \mathbf{y}}{\partial \theta}\right)^{T} \mathbf{V}^{-\mathbf{1}}\left(\frac{\partial \mathbf{y}}{\partial \theta}\right)\right)^{-1}
$$

where $p$ is the number of estimators, $\mathbf{y}$ is a vector of outputs, and $\mathbf{V}$ is the diagonal variance matrix of measurement errors. The correlation matrix, a normalized symmetric matrix which approximates the correlation between parameters, 
is calculated from the covariance matrix (Eq. (2)) as

$$
\operatorname{cor}\left(\theta_{i}, \theta_{k}\right)=\frac{\operatorname{Cov}(\hat{\theta})}{\sqrt{\operatorname{diag}(\operatorname{Cov}(\hat{\theta})) \operatorname{diag}(\operatorname{Cov}(\hat{\theta}))}}
$$

For large $N$ the $100(1-\alpha) \%$ confidence interval of the parameters can be approximated by

$$
\hat{\theta} \pm t_{N-p}^{\alpha / 2} \sqrt{\operatorname{diag}(\operatorname{Cov}(\hat{\theta}))}
$$

where $t_{N-p}^{\alpha / 2}$ is the student's t-distribution corresponding to the $\alpha / 2$ percentile and with $N-p$ degrees of freedom. In Eq. (4) it is implicitly assumed that the various parameters are independent of each other. In reality the parameters are correlated resulting in confidence ellipsoids, or hyper-ellipsoids.

\subsubsection{Bootstrap technique for uncertainty of parameter estimators}

The LSQ method is by far the most well-known approach for parameter estimation. There are, however, several alternative parameter estimation methods. One of these is a method called bootstrap [29]. The basic idea of the bootstrap method is that it relies on random sampling with replacement of the residuals, to generate a number of synthetic pseudo-experimental data sets. Since the development of the method by Efron [29] several bootstrapping schemes have been suggested. In this work we employ one of the variants used for regression problems, namely re-sampling of residuals. This scheme consists of four main steps;

Step 1 Input parameters are correlated to the experimental data using Eq. (1) as the objective function.

Step 2 Residuals from the correlation are randomly sampled (with replacement) and added to the previously correlated output values.

Step 3 The parameters are refitted to the new synthetic data. Step 2 and 3 is repeated a large number of times to simulate repeated experimental runs.

Step 4 The distribution of regressed mean estimators are evaluated to obtain confidence regions and parameter correlations. 
If the underlying distribution of errors is close to the normal distribution, bootstrapping usually gives results similar to the LSQ approach. An advantage of bootstrapping, however, is that it does not make any assumption with respect to the underlying distribution of errors [42].

\subsection{Propagation of Parameter Uncertainty to Model Predictions}

Uncertainty associated with the predictions from thermodynamic models can generally be classified as; (a) input uncertainty and (b) structural uncertainty (or model error). The structural uncertainty deals with the mathematical form of the EoS, since all models, no matter their complexity, are only an approximation of the real physical system. The input uncertainty, on the other hand, represents the uncertainty in adjustable parameters mainly due to uncertainty in the experimental data, and the method employed to find these parameters $[13,43]$. The uncertainty in adjustable parameters will propagate through the model and will affect the accuracy of model outputs.

One popular method used for error propagation is Monte Carlo analysis. The Monte Carlo analysis is based on multiple model evaluations using inputs sampled from their corresponding uncertainty which is usually described by a certain distribution function (uniform, normal, etc.). The main advantage of the Monte Carlo procedure is that uncertainty results can be obtained directly from the model in question without the need for calculation of the Jacobian matrix which is required by linear error propagation. It is thus conceptually easy to implement and, perhaps most importantly for this work, it can be used to propagate uncertainties through a sequence of models without the need for modifications to the original model.

\subsubsection{A Monte Carlo uncertainty analysis}

For notational convenience assume that the desired output property from any of the equations of state under investigation can be represented by a function of the form

$$
\mathbf{y}=\mathbf{f}(\theta, \mathbf{n}, P, T)
$$


where $\mathbf{y}$ is a vector of output model predictions, $\theta$ is a vector of model inputs and $\mathbf{n}, P$ and $T$ are the composition vector, pressure and temperature respectively. The expression in Eq. (5) may represent not only the equation of state but a sequence of linked models or expressions needed to calculate a desired output property. For instance $f$ may represent a dew or bubble point calculation.

Monte Carlo uncertainty analysis is based on performing multiple model evaluations of a function of the form in Eq. (5) with inputs sampled from a probabilistic distribution. The Monte Carlo uncertainty analysis involves four steps [43]:

Step 1 Specification of the range and underlying probability distribution of the input variables.

Step 2 Sampling from the input range and distribution specified in the first step, to simulate parameters obtained from repeated experimental runs.

Step 3 Evaluation of Eq. (5) for each input sample.

Step 4 Representation and interpretation of results.

In this work we employ the (kernel) probability density functions of the input variables as estimated from the bootstrapped subsamples. Commonly used methods to take samples from the input space in step 2 are; random sampling, shifted Hammersley sampling [44], equal probability sampling [13] and LHS [45]. In this work the samples are chosen using LHS sampling. Input parameter correlation is induced by applying the Iman-Conover correlation control method [46]. Additional introductions to Monte Carlo analysis are available elsewhere e.g. $[47-50]$ and references herein.

\section{Results and Discussion}

\subsection{Parameter estimation - uncertainty and correlation}

The pure compound parameters of approaches A-F were correlated to the experimental saturated liquid density and saturated vapor pressures using Eq. (1) 

used for the saturated liquid density (below $295 \mathrm{~K}$ the experimental uncertainty is estimated to less than $\pm 0.015 \%)$. For the saturated vapor pressure we employ data from [51-53] (experimental uncertainty estimated to $\pm 0.016-0.012 \%$, $\pm 100 \mathrm{~Pa}$ and $\pm 0.1 \%$ respectively). It is assumed that the standard deviation, $\sigma_{i}^{e x p}$, of each experimental point can be approximated by the experimental uncertainties given in the references.

\subsubsection{Least Squares estimation}

Table 2 summarizes the estimated parameters for each modeling approach. We note that, as expected from the work of Tsivintzelis et al. [28] and Bjørner and Kontogeorgis [33], excellent agreement to saturated vapor pressures and saturated liquid densities are obtained for all approaches. With the exception of association scheme $2 \mathrm{~B}$ the parameters for approach A-D are similar to those presented by Tsivintzelis et al. [28]. The small difference in parameters can probably be explained by the slightly different objective function and the use of experimental data for the correlation, rather than pseudo-experimental data. The deviations generally decrease when $\mathrm{CO}_{2}$ is treated as either an associating or a quadrupolar compound. For approaches with four or five parameters the excellent correlations, however, may simply be due to the added flexibility of additional model terms and parameters. The good correlations which are already obtained for the approaches using only three adjustable parameters (A and E) suggest that models with more parameters are over-parameterized, which in turn reduces the reliability of the obtained parameters (see tables 4 and 5).

Tables 3-6 show the estimated parameter uncertainty, the correlation matrix between the parameters and the mean estimate for modeling approaches $\mathrm{B}, \mathrm{D}$, $\mathrm{E}$ and $\mathrm{F}$. The confidence intervals are presented as a percentage of its mean estimator. Only the lower triangular part of the symmetric correlation matrix is shown.

It can be seen from table 3 that small confidence intervals are obtained as well as low correlations between $b_{0}$ and both $\Gamma$ and $c_{1}$ for approach E. A 
Table 2: Correlated pure compound parameters and \%AADs in saturated liquid density and saturated pressure for $\mathrm{CO}_{2}$ with the CPA EoS (Approaches A-D) and the qCPA (Approaches E-F). The parameters are correlated in the temperature range $T_{r}=0.7-0.9$.

\begin{tabular}{cccccccccc}
\hline \multirow{2}{*}{ Designation } & Approach & $\begin{array}{c}b_{0} \\
\mathrm{~mL} / \mathrm{mol}\end{array}$ & $\mathrm{K}\left(a_{0} /\left(R b_{0}\right)\right.$ & $c_{1}$ & $\beta \cdot 1000$ & $\epsilon / R$ & $b_{0}^{Q}$ & \multicolumn{3}{c}{ \%AAD $^{a}$} \\
& & - & - & $\mathrm{K}$ & $\mathrm{mL} / \mathrm{mol}$ & in $P^{s a t}$ & in $\rho^{l i q}$ \\
\hline A & n.a. & 27.3 & 1550 & 0.77 & - & - & - & 0.18 & 0.95 \\
B & 2B & 26.9 & 1145 & 0.43 & 42.3 & 1089 & - & 0.07 & 0.11 \\
C & 3B & 28.1 & 1310 & 0.64 & 34.7 & 671 & - & 0.06 & 0.10 \\
D & $4 \mathrm{C}$ & 28.4 & 1329 & 0.66 & 25.7 & 513 & - & 0.07 & 0.10 \\
E & Quad & 27.9 & 1284 & 0.68 & - & - & - & 0.13 & 0.46 \\
F & Quad & 28.5 & 1027 & 0.60 & - & - & 20.2 & 0.12 & 0.07 \\
\hline
\end{tabular}

${ }_{a} \% A A D=\frac{100}{N^{\exp }} \sum_{i}^{N^{\exp }}\left|\frac{x_{i}^{\text {calc }}-x_{i}^{\text {exp }}}{x_{i}^{\exp }}\right|$ where $x$ stands for $P^{\text {sat }}$ or $\rho^{l i q}$ and $N^{\exp }$ is the number of experimental data.

Table 3: Estimated $\mathrm{CO}_{2}$ parameters, uncertainty as a $95 \%$ confidence interval (CI) in percent of the parameter estimate, and parameter correlation matrix when modeling approach $\mathbf{E}$ is employed.

\begin{tabular}{cccccc}
\hline & & & \multicolumn{4}{c}{ Correlation matrix } \\
$\boldsymbol{\theta}$ & Estimator & $95 \%$ CI $(\%)$ & $b_{0}$ & $\Gamma$ & $c_{1}$ \\
\hline$b_{0}$ & 27.9 & 0.08 & 1 & & \\
$\Gamma$ & 1284 & 0.13 & 0.29 & 1 & \\
$c_{1}$ & 0.68 & 0.77 & -0.01 & -0.95 & 1 \\
\hline
\end{tabular}



for the slightly larger confidence interval of the $c_{1}$ parameter. The correlation is unsurprising considering that these two parameters are closely related in the attractive Soave-Redlich-Kwong (SRK) term (see Eq. (A.5) in Appendix A). Similar results are obtained when $\mathrm{CO}_{2}$ is modelled as an inert (approach A).

Table 4: Estimated $\mathrm{CO}_{2}$ parameters, uncertainty as a $95 \%$ confidence interval (CI) in percent of the parameter estimate, and parameter correlation matrix when modeling approach $\mathbf{F}$ is employed.

\begin{tabular}{ccccccc}
\hline & & \multicolumn{5}{c}{ Correlation matrix } \\
$\boldsymbol{\theta}$ & Mean estimator & $95 \%$ CI $(\%)$ & $b_{0}$ & $\Gamma$ & $c_{1}$ & $b_{0}^{Q}$ \\
\hline$b_{0}$ & 28.5 & 0.09 & 1 & & & \\
$\Gamma$ & 1027 & 1.19 & -0.98 & 1 & & \\
$c_{1}$ & 0.60 & 0.63 & -0.91 & 0.91 & 1 & \\
$b_{0}^{Q}$ & 20.2 & 1.15 & -0.98 & 0.99 & 0.92 & 1 \\
\hline
\end{tabular}

The results for approach $\mathrm{F}$, shown in table 4, indicate that when one additional adjustable parameter is added to the model, all model parameters become highly correlated. That is, a small change in one parameter can be compensated by a change in another parameter. This suggests that the model is over-parametrized making it difficult, if not impossible, to uniquely identify its parameters. The large correlations, however, have not increased the estimated confidence intervals significantly, which may suggest that the parameters are also highly sensitive. This may indicate that such model extensions are undesirable, at least when it comes to parameter estimation, even if they have the potential to improve model predictions.

When $\mathrm{CO}_{2}$ is modelled as an associating species (approaches B-D) there are even more adjustable parameters. Due to the added flexibility from the parameters, we would also expect the approaches with association to be highly correlated. It turns out, however, that the degree of correlation depends very much on the chosen association scheme. Tables 5 and 6 show the confidence 
Table 5: Estimated $\mathrm{CO}_{2}$ parameters, uncertainty as a $95 \%$ confidence interval (CI) in percent of the parameter estimate, and parameter correlation matrix when modeling approach $\mathbf{D}$ is employed.

\begin{tabular}{cccccccc}
\hline & & & \multicolumn{7}{c}{ Correlation matrix } \\
$\boldsymbol{\theta}$ & Mean estimator & $95 \%$ CI $(\%)$ & $b_{0}$ & $\Gamma$ & $c_{1}$ & $\beta$ & $\epsilon$ \\
\hline$b_{0}$ & 28.4 & 0.06 & 1 & & & & \\
$\Gamma$ & 1329 & 0.79 & -0.47 & 1 & & & \\
$c_{1}$ & 0.66 & 4.96 & -0.24 & -0.69 & 1 & & \\
$\beta$ & 25.7 & 22.65 & -0.10 & -0.80 & 0.97 & 1 & \\
$\epsilon$ & 512.7 & 9.63 & 0.26 & 0.68 & -0.99 & 0.98 & 1 \\
\hline
\end{tabular}
are obtained with only three adjustable parameters, we suspect that the latter possibility is predominant. However, the model structure clearly matters a 
great deal, as the identifiability problems are much more significant when the $2 \mathrm{~B}$ association scheme is employed rather than the $4 \mathrm{C}$ or $3 \mathrm{~B}$ association schemes. This indicates that the $2 \mathrm{~B}$ scheme is less suited to model $\mathrm{CO}_{2}$ than the $4 \mathrm{C}$ and 3B scheme. It is interesting to note that Tsivintzelis et al. [28] arrived at the same conclusion by evaluating the phase equilibria of a large number of binary mixtures.

Table 6: Estimated $\mathrm{CO}_{2}$ parameters, uncertainty as a $95 \%$ confidence interval (CI) in percent of the parameter estimate, and parameter correlation matrix when modeling approach $\mathbf{B}$ is employed.

\begin{tabular}{rccccccc}
\hline & & & \multicolumn{5}{c}{ Correlation matrix } \\
$\boldsymbol{\theta}$ & Mean estimator & $95 \%$ CI $(\%)$ & $b_{0}$ & $\Gamma$ & $c_{1}$ & $\beta$ & $\epsilon$ \\
\hline$b_{0}$ & 26.9 & 3.33 & 1 & & & & \\
$\Gamma$ & 1145 & 10.53 & 0.99 & 1 & & & \\
$c_{1}$ & 0.43 & 38.27 & 0.99 & 0.99 & 1 & & \\
$\beta$ & 42.3 & 16.82 & 0.97 & 0.97 & 0.99 & 1 & \\
$\epsilon$ & 1089 & 19.53 & -0.99 & -0.99 & 0.99 & 0.99 & 1 \\
\hline
\end{tabular}

\subsubsection{Parameters from the Bootstrap method}

The bootstrap method for parameter estimation gives a distribution of parameter sets generated by the, slightly, different synthetic data sets. Figure 1 shows the obtained distribution of input parameters for approach $\mathrm{E}$ as a histogram, with the number of occurences on the left y-axis, and as the estimated probability density function (right y-axis). From figure 1 we see that the distributions of all input parameters follow a normal distribution quite accurately, and we would expect the mean value of the input parameters to be similar to those obtained from LSQ. Indeed by comparing figure 1 with table 3 we see that the mean input parameters are almost identical to those obtained from the LSQ estimation. The main difference is, that distributions from bootstrapping are somewhat wider than the confidence intervals obtained from LSQ estimation. 

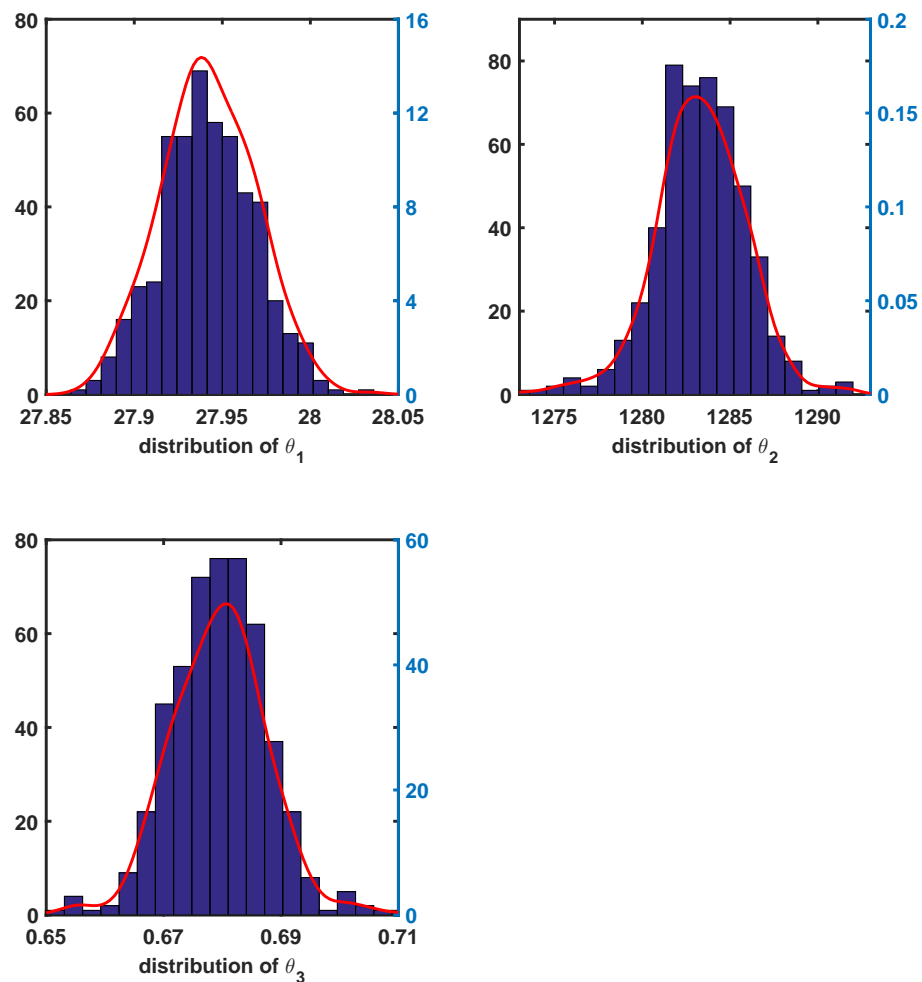

Figure 1: Histograms approximating the distribution of each parameter (left y-axis), obtained from 500 re-sampled bootstraps, using modeling approach $\mathbf{E}$ for $\mathrm{CO}_{2}$. The full red lines show the estimated probability density function (right y-axis). 

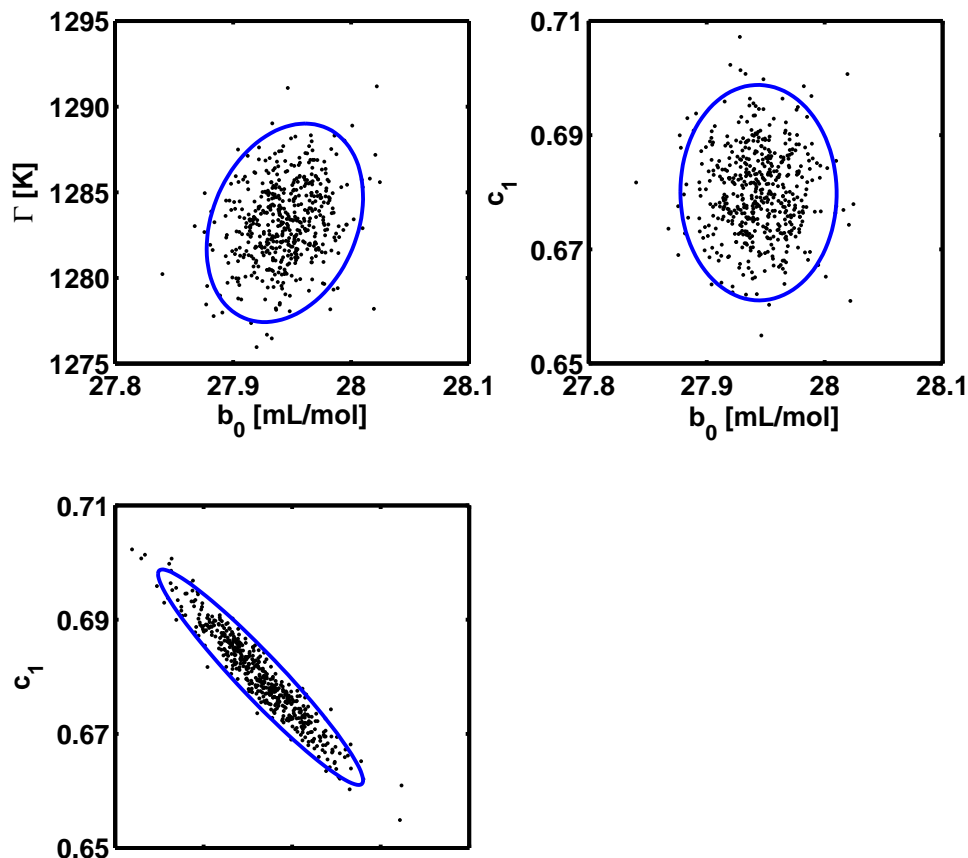

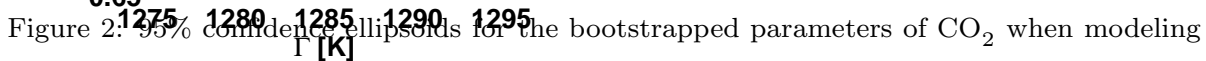
approach $\mathbf{E}$ is employed. Each dot represents a realized parameter combination and each subfigures represents the dependency (if any) of one parameter on another.

Figure 2 visualizes the correlation between certain parameters by plotting each input parameter as a function of another input parameter. The ellipsoids in figure 2 represent $95 \%$ confidence intervals. The closer an ellipsoid is to a circle the more random is the parameter pair, and thus the weaker the correlation between the two. It is clear from the figure that there is almost no correlation between $\Gamma$ and $b_{0}$ and $c_{1}$ and $b_{0}$, while the value of $\Gamma$ and $c_{1}$ depend on each other. This is consistent with the results in table 3 for LSQ estimation. Almost exactly the same conclusion can be made when approach A is employed. Both modeling approaches employ only three adjustable parameters.

Figures 3 and 4 show the bootstrapping results with approach F It is clear from figure 3 that the input parameter distributions are no longer normal, rather it looks like the distribution of all input parameters are bimodal. That is, there 
are two distinct peaks, or modes, in the distributions. This may suggest that tributions. Although not shown both LSQ estimation and bootstrapping tend to agree about the degree of correlation between parameters. 

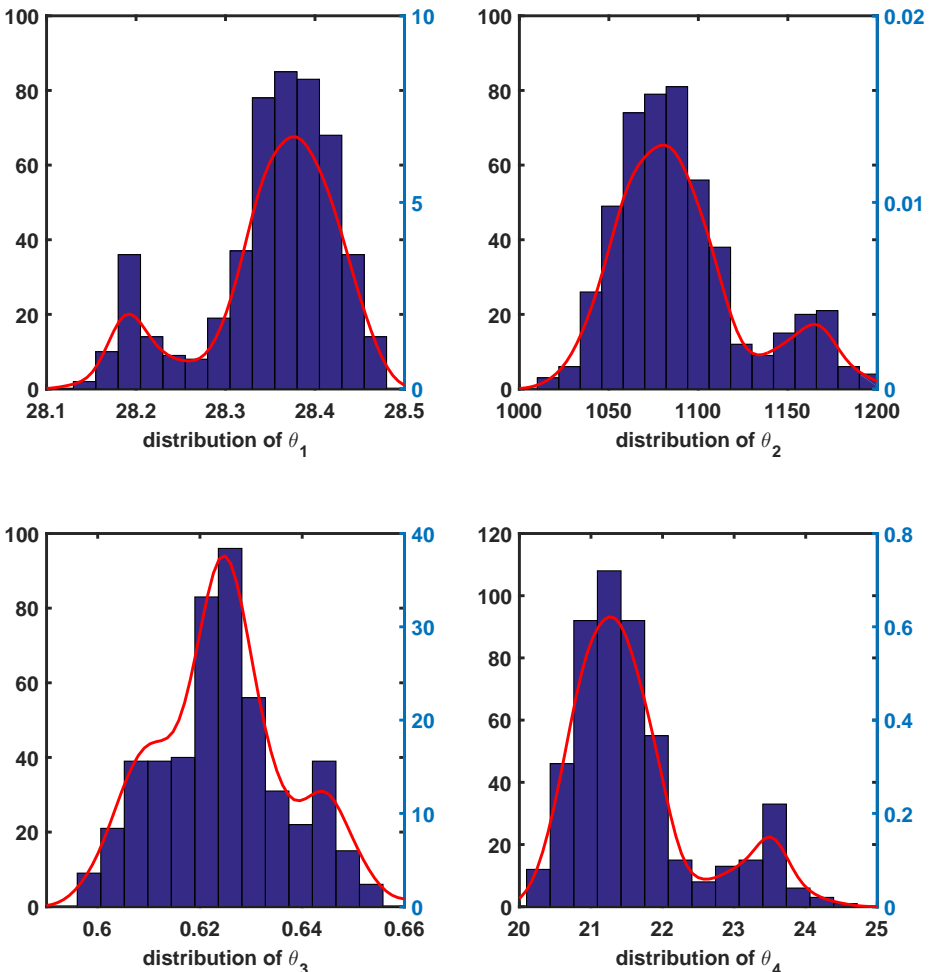

Figure 3: Histograms approximating the distribution of each parameter (left y-axis), obtained from 500 re-sampled bootstraps, using modeling approach $\mathbf{F}$ for $\mathrm{CO}_{2}$. The full red lines show the estimated probability density function (right y-axis). 

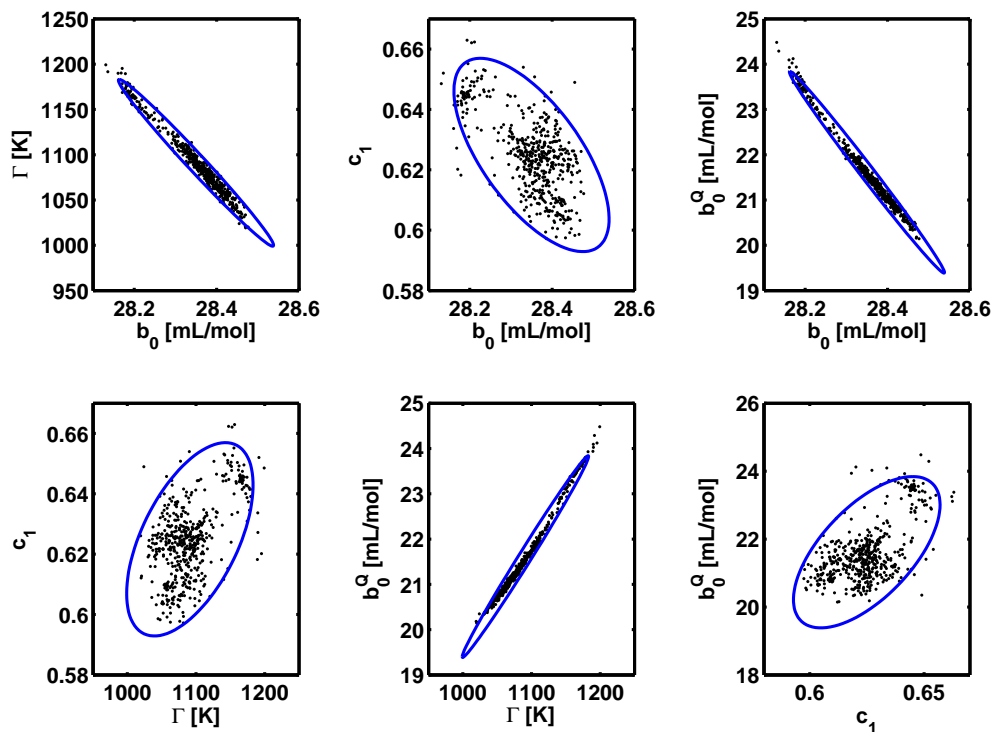

Figure 4: $95 \%$ confidence ellipsoids for the bootstrapped parameters of $\mathrm{CO}_{2}$ when modeling approach $\mathbf{F}$ is employed. Each dot represents a realized parameter combination and each subfigures represents the dependency (if any) of one parameter on another. 

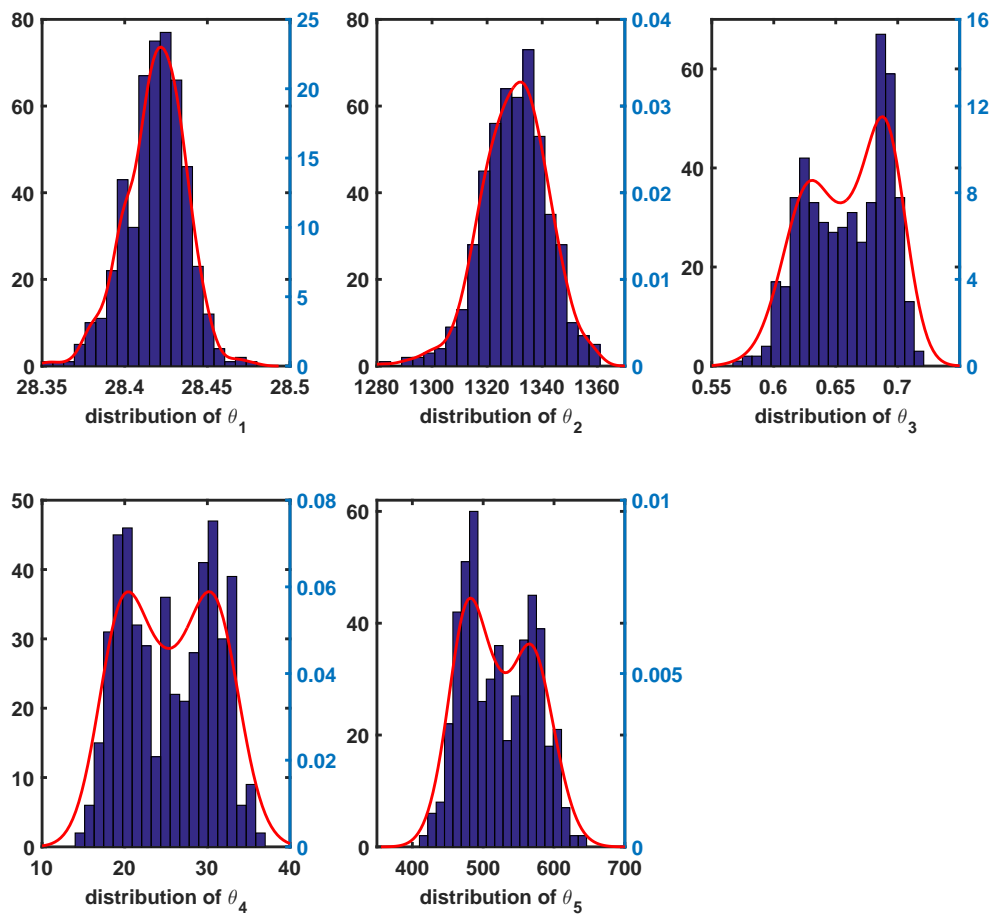

Figure 5: Histograms approximating the distribution of each parameter (left y-axis), obtained from 500 re-sampled bootstraps, using modeling approach $\mathbf{D}$ for $\mathrm{CO}_{2}$. The full red lines show the estimated probability density function (right y-axis). 


\subsection{Propagation of parameter estimation errors to property prediction}

parameter sets does not incur uncertainty in other outputs, which may depend on the inputs in a different way.

It is well-known that the isochoric heat capacity is a challenging property to predict even with modern equations of state $[33,56]$. It is believed that this 335 is primarily due to structural issues with the temperature dependence of the equations of state. It is possible, however, that at least part of the problem can be explained by uncertainties in the parameters. Figures 7 and 8 map the input uncertainty onto the residual isochoric and isobaric heat capacity at saturation respectively. From both plots we see that the uncertainty is significant 


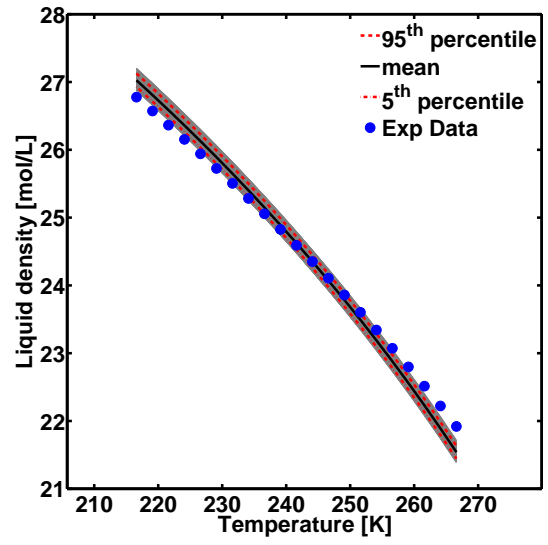

(a) Approach $\mathbf{A}$.

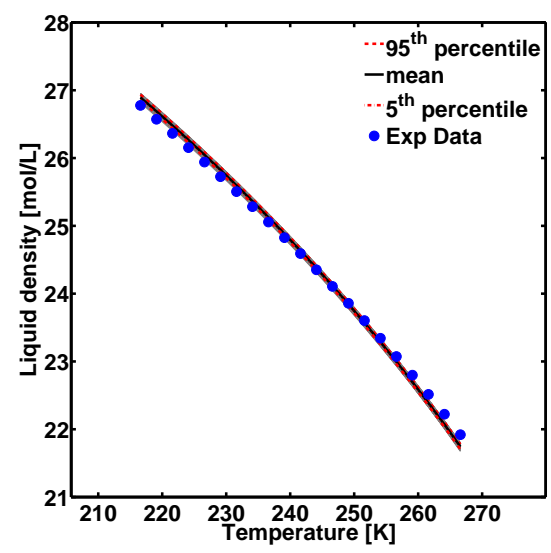

(c) Approach E.

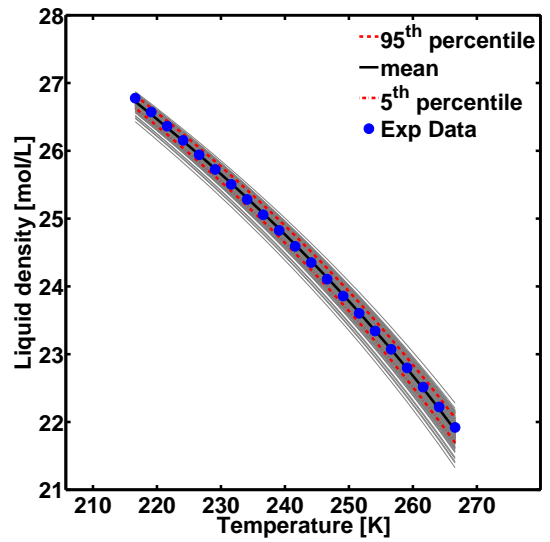

(b) Approach D.

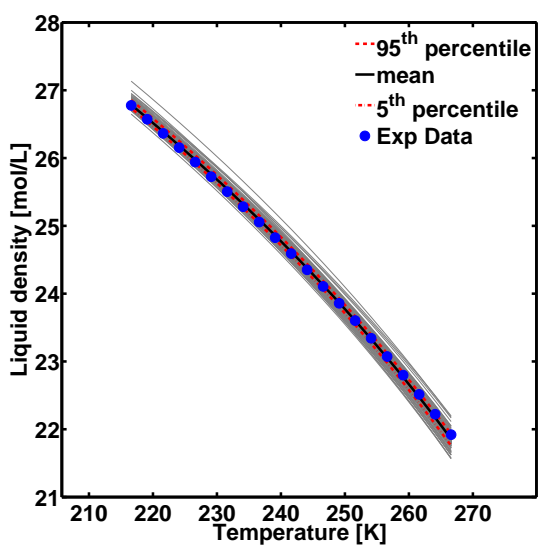

(d) Approach F.

Figure 6: Propagated uncertainty in the model predictions for the liquid density at saturation. Approach A (a), D (b), E (c) and F (d) is employed. Grey lines represent the simulations, red dashed lines are the 5th and 95th percentile of the simulations and black full lines are the mean of the simulations. As the simulations, and their mean are almost identical the lines are difficult to see. Blue circles are pseudo-experimental data from Span and Wagner [30]. 
for approach D, smaller, but still present, for approaches $\mathrm{E}$ and $\mathrm{F}$ and almost non-existent for approach A. This illustrates how larger uncertainty in input parameters, e.g. for the approaches using more adjustable parameters, result in higher uncertainties in the non-fitted derivative properties. The large uncertainties for the isochoric heat capacity in figure $7 \mathrm{~b}$ suggest that the uncertainty in pure compound parameters lead to significant uncertainties in the temperature derivatives of the equation of state. This should be non-surprising as the three most uncertain parameters in approach $\mathrm{D}$ are parameters responsible for the temperature dependence $\left(c_{1}, \varepsilon, \beta\right)$ (Eq. (A.5) and (A.8)). This may, in part, explain why $C_{V}^{r e s}$ is such a difficult property to calculate for many equations of state $[33,56]$. However, the deviation from the pseudo-experimental data is so large, that at least part of the deviation is expected to be due to structural uncertainty rather than input uncertainty. It is somewhat surprising that CPA with $\mathrm{CO}_{2}$ as an inert molecule, models $C_{V}^{r e s}$ so well, while none of the more sophisticated equations of state yield particularly good results.

55 Due to the relation between $C_{V}^{r e s}$ and $C_{P}^{r e s}$ (see Appendix B) uncertainties in $C_{V}^{r e s}$ should lead to, at least, similar sized uncertainties in $C_{P}^{r e s}$. The uncertainty in figure 8 is of the same magnitude as that in figure 7 , which suggests that the input uncertainty does not lead to significant additional uncertainties in the ratio between the $(\partial P / \partial T)^{2}$ and $\partial P / \partial V$ derivatives. As opposed to the results for $C_{V}^{r e s}$ we see that the more advanced approaches all estimate $C_{P}^{r e s}$ rather well within the input uncertainty, while the calculations when $\mathrm{CO}_{2}$ is treated as an inert compound fail to follow the trend of the pseudo-experimental data. We may note, however, that while approaches D, E and F capture the right trend of $C_{P}^{r e s}$ as a function of temperature, the only reason that the predictions represent the experimental data so well, is the off-set cause by the poor representation of $C_{V}^{r e s}$, thus some cancellation of error must occur between $C_{V}^{r e s}$ and the ratio $(\partial P / \partial T)^{2} / \partial P / \partial V$.

One of the primary objectives of most equations of state is the accurate description of phase equilibria $[56,57]$. It is therefore of particular interest 70 to investigate how the input uncertainty affects the prediction of vapour liq- 


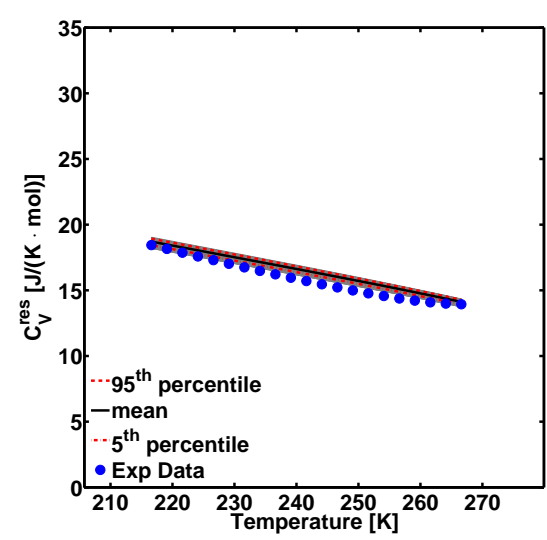

(a) Approach A.

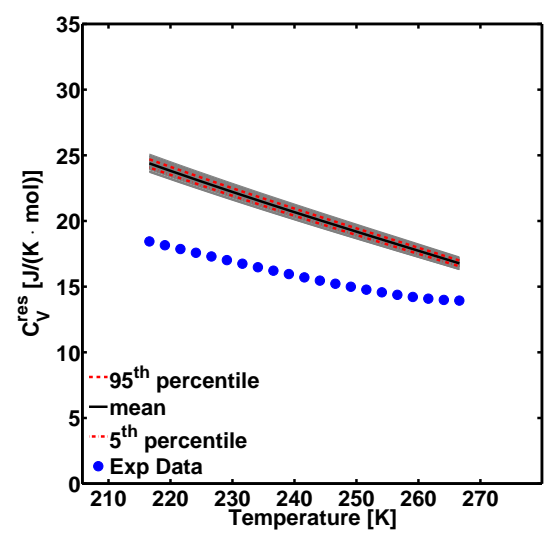

(c) Approach E.

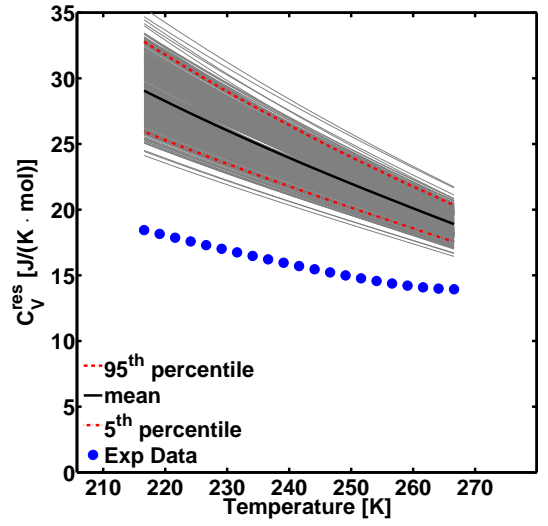

(b) Approach D.

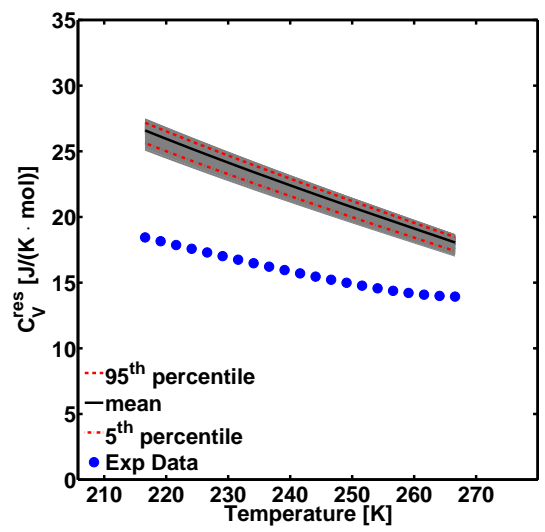

(d) Approach F.

Figure 7: Propagated uncertainty in the model predictions for the residual isochoric heat capacity of $\mathrm{CO}_{2}$ at saturation, employing approach $\mathbf{A}(\mathrm{a}), \mathbf{D}(\mathrm{b}), \mathbf{E}$ (c) and $\mathbf{F}$ (d). Grey lines represent the simulations, red dashed lines are the 5th and 95th percentile of the simulations and black full lines are the mean of the simulations. Pseudo-experimental data from Span and Wagner [30]. 


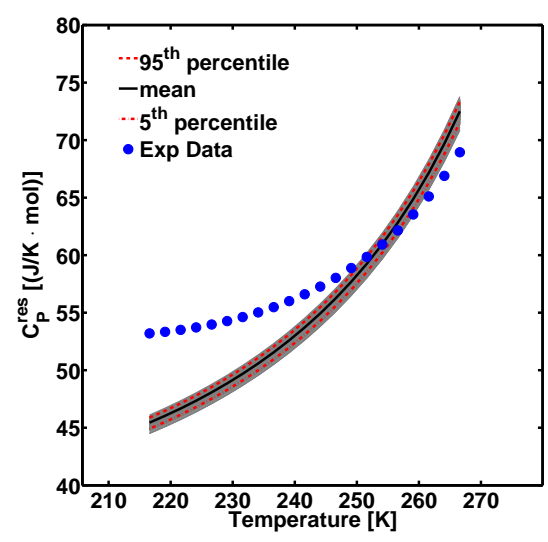

(a) Approach A.

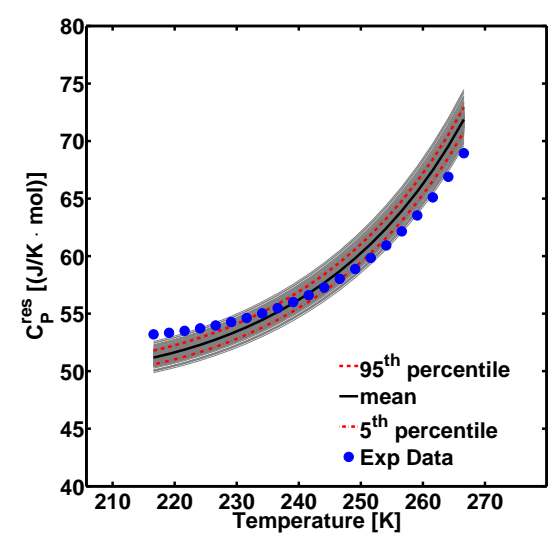

(c) Approach E.

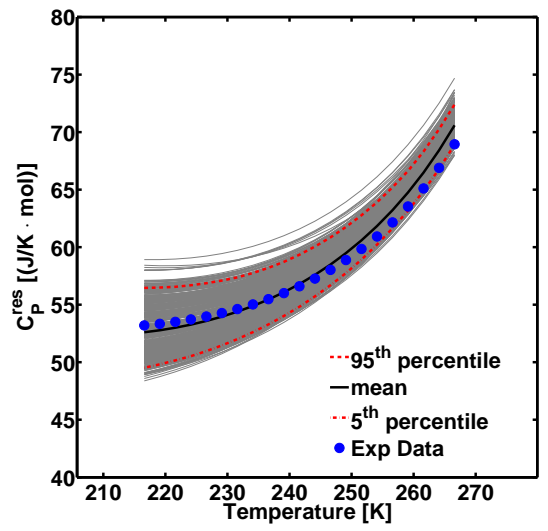

(b) Approach $\mathbf{D}$.

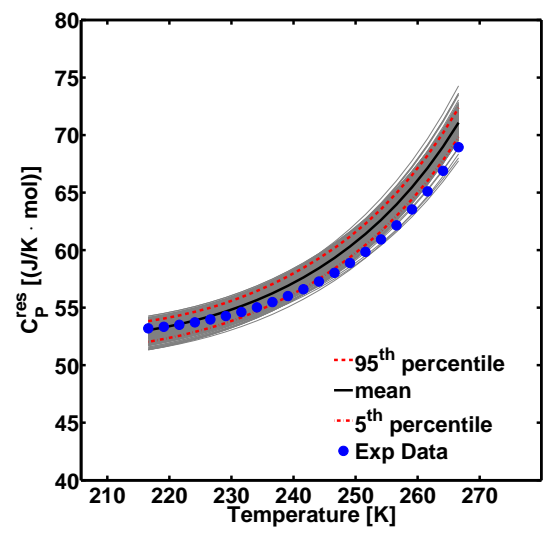

(d) Approach F.

Figure 8: Propagated uncertainty in the model predictions for the residual isobaric heat capacity of $\mathrm{CO}_{2}$ at saturation, employing approach $\mathbf{A}$ (a), $\mathbf{D}(\mathrm{b}), \mathbf{E}$ (c) and $\mathbf{F}$ (d). Grey lines represent the simulations, red dashed lines are the 5th and 95th percentile of the simulations and black full lines are the mean of the simulations. Pseudo-experimental data from Span and Wagner [30]. 
uid equilibrium (VLE). In this work we only take the input uncertainty in the $\mathrm{CO}_{2}$ parameters into account, and ignore the input uncertainty of the other component. The mixtures investigated are two $\mathrm{CO}_{2}+$ hydrocarbon mixtures.

Hydrocarbons are modelled with only three parameters (approach A) and the 375 predictions of binary hydrocarbon+hydrocarbon mixtures, are generally quite accurate. It is thus expected, that the error in their parameters are negligible, and that almost all sources of error in mixtures of $\mathrm{CO}_{2}$ and hydrocarbons are due to the quadrupolar $\mathrm{CO}_{2}$ molecule.

Figures 9-10 show the propagated input uncertainty on the $\mathrm{CO}_{2}$ +ethane and $\mathrm{CO}_{2}+$ propane VLEs at $250 \mathrm{~K}$ and $230 \mathrm{~K}$ respectively. The small quadrupolar moment of ethane is ignored. All VLE plots are predictions $\left(k_{i j}=0\right)$. The uncertainty with approaches A and E, both of which have three input parameters, is negligible. The model error with approach A, however, is significant, illustrating the need for improved modeling approaches. All other model approaches qualitatively predict the azeotrope in figure 9 and improve the representation of the $\mathrm{CO}_{2}+$ propane VLE.

The output uncertainty with approach D for the VLE is quite small, which is in contrast to the uncertainties in the pure compound heat capacities. On the other hand, while uncertainties in the pure compound derivative properties were small to moderate for approach F, they are clearly significant for the liquid phase of the VLE systems. The uncertainty depends on the mole fraction of $\mathrm{CO}_{2}$, with the largest uncertainty in model output being around $x_{\mathrm{CO}_{2}}=0.5$. It is noteworthy that while the uncertainties in the VLE systems are significant for approach $\mathrm{F}$, it is the only model which could accurately predict the data within its $95 \%$ percentile.

In any case, the results show that for models such as the qCPA and CPA with association a simple LSQ estimation may not give the optimal parameters in terms of phase equilibrium predictions, as the input uncertainty results in significant output uncertainties. It is worth noting that it is the same parameter set which generate the best (closest to the the experimental data) prediction in figures 9-10. It is thus possible to find a parameter set, which generates 
excellent predictions for the VLE of $\mathrm{CO}_{2}+$ alkanes, based only on uncertainties in the input parameters. 


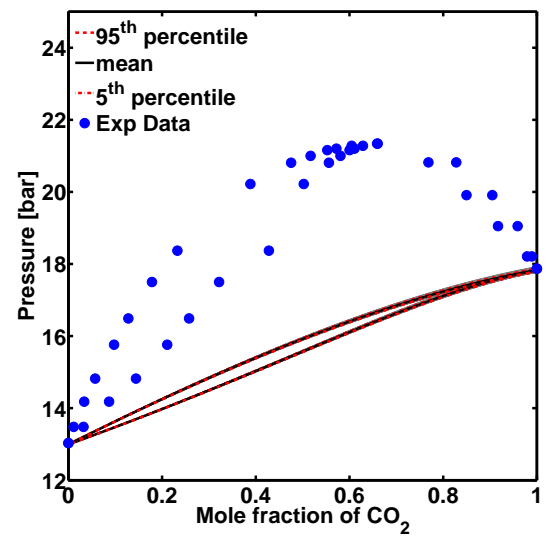

(a) Approach $\mathbf{A}$.

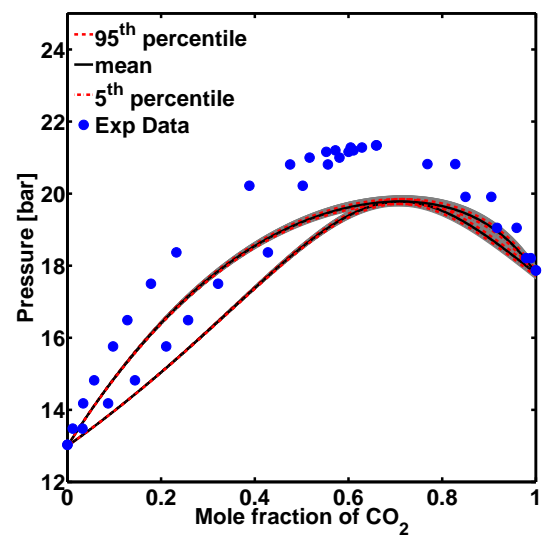

(c) Approach E.

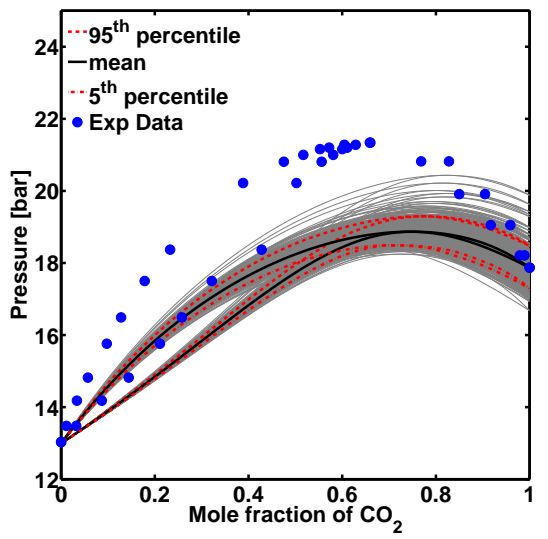

(b) Approach D.

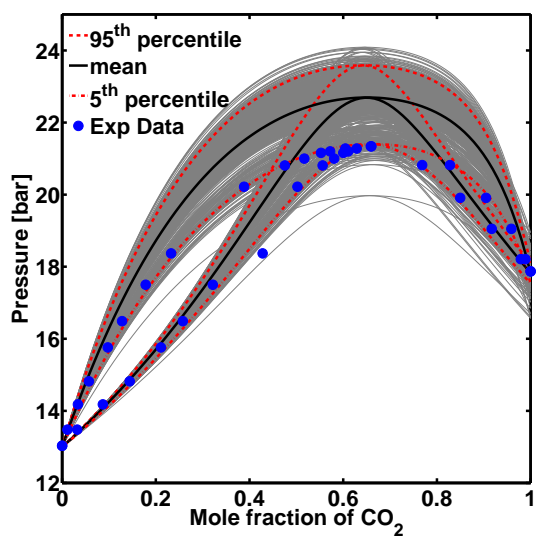

(d) Approach F.

Figure 9: Propagated uncertainty in the model predictions for the $\mathrm{CO}_{2}$ +ethane VLE at $\mathrm{T}=250 \mathrm{~K}$. Employing approach A (a), D (b), E (c) and F (d). Grey lines represent the simulations, red dashed lines are the 5th and 95th percentile of the simulations and black full lines are the mean of the simulations. Experimental data from [58]. 


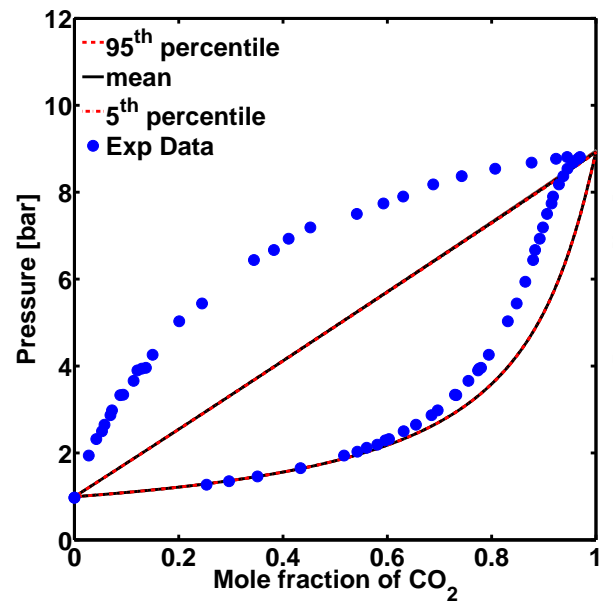

(a) Approach $\mathbf{A}$.

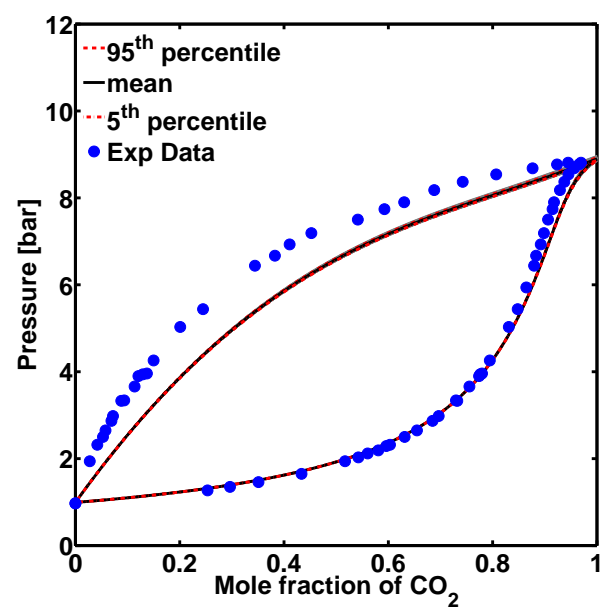

(c) Approach E.

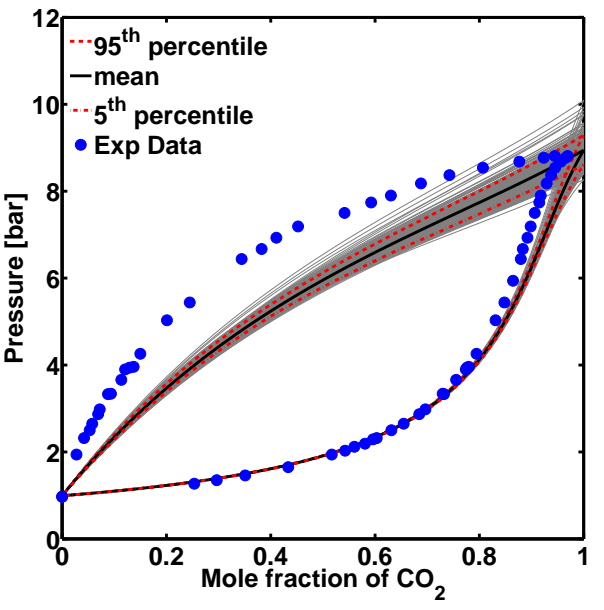

(b) Approach D.

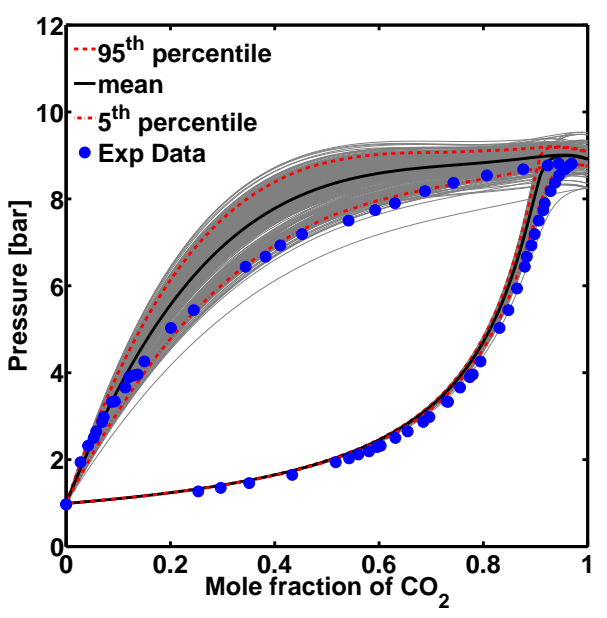

(d) Approach F.

Figure 10: Propagated uncertainty in the model predictions for the $\mathrm{CO}_{2}+$ propane VLE at $\mathrm{T}=230 \mathrm{~K}$. Employing approach $\mathbf{A}$ (a), D (b), E (c) and $\mathbf{F}$ (d). Grey lines represent the Monte Carlo simulations, red dashed lines are the 5th and 95th percentile of the simulations and black full lines are the mean of the simulations. Experimental data from [59]. 


\subsection{The effect of adding additional output properties}

in the VLE estimation is still high for approach F. It is furthermore noteworthy that approach $\mathrm{F}$ is, again, the only model which can predict the data within its 95th percentile. The uncertainty range has, however, changed so that the vari- 

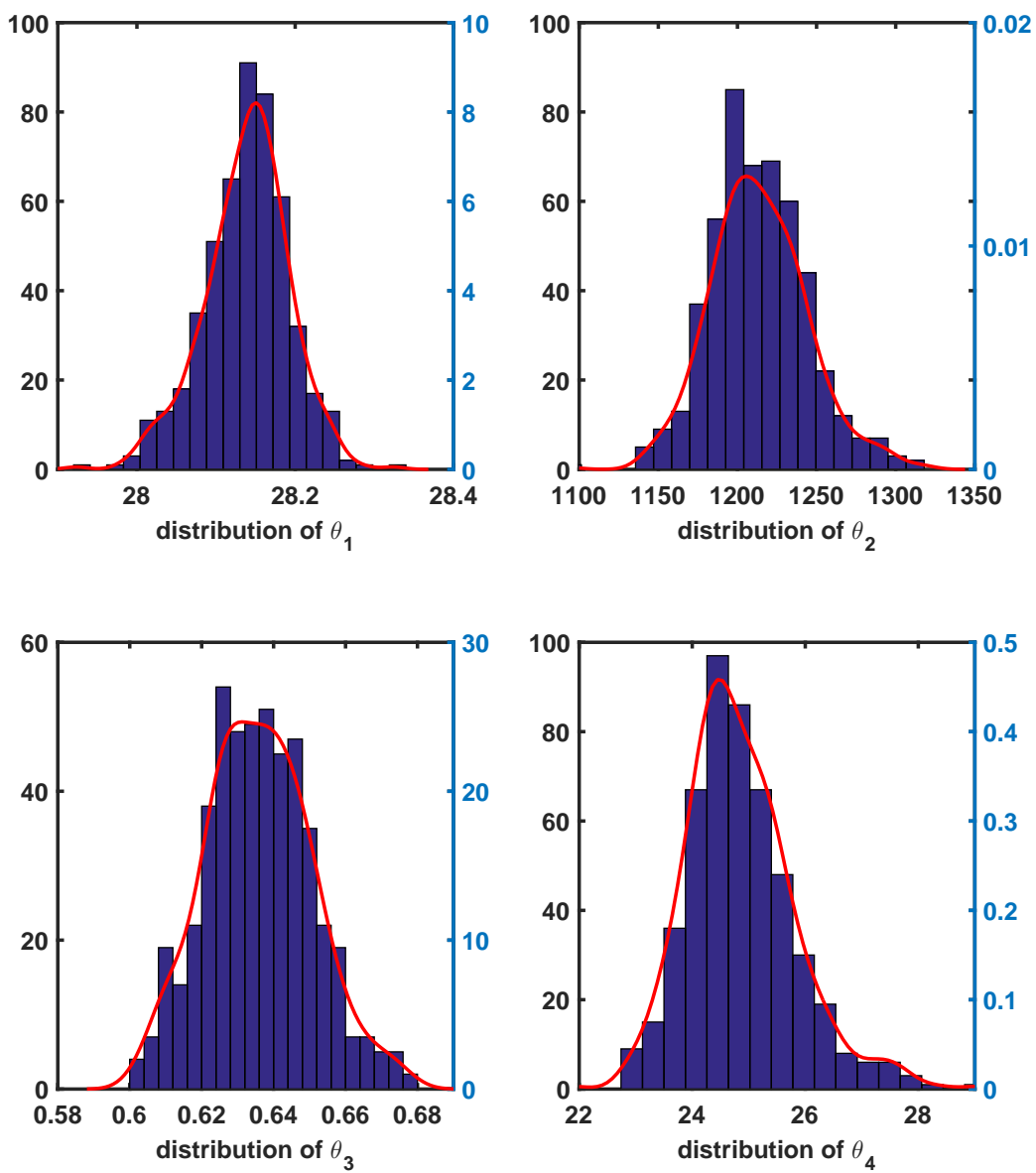

Figure 11: Histograms approximating the distribution of each parameter (left y-axis), obtained from 500 re-sampled bootstraps, using modeling approach $\mathbf{F}$ for $\mathrm{CO}_{2}$. The parameters have been fitted to $\Delta H^{v a p}$ in addition to $\rho_{\text {sat }}^{\text {liq }}$ and $P^{s a t}$. The full red line shows the estimated probability density function (right y-axis). 

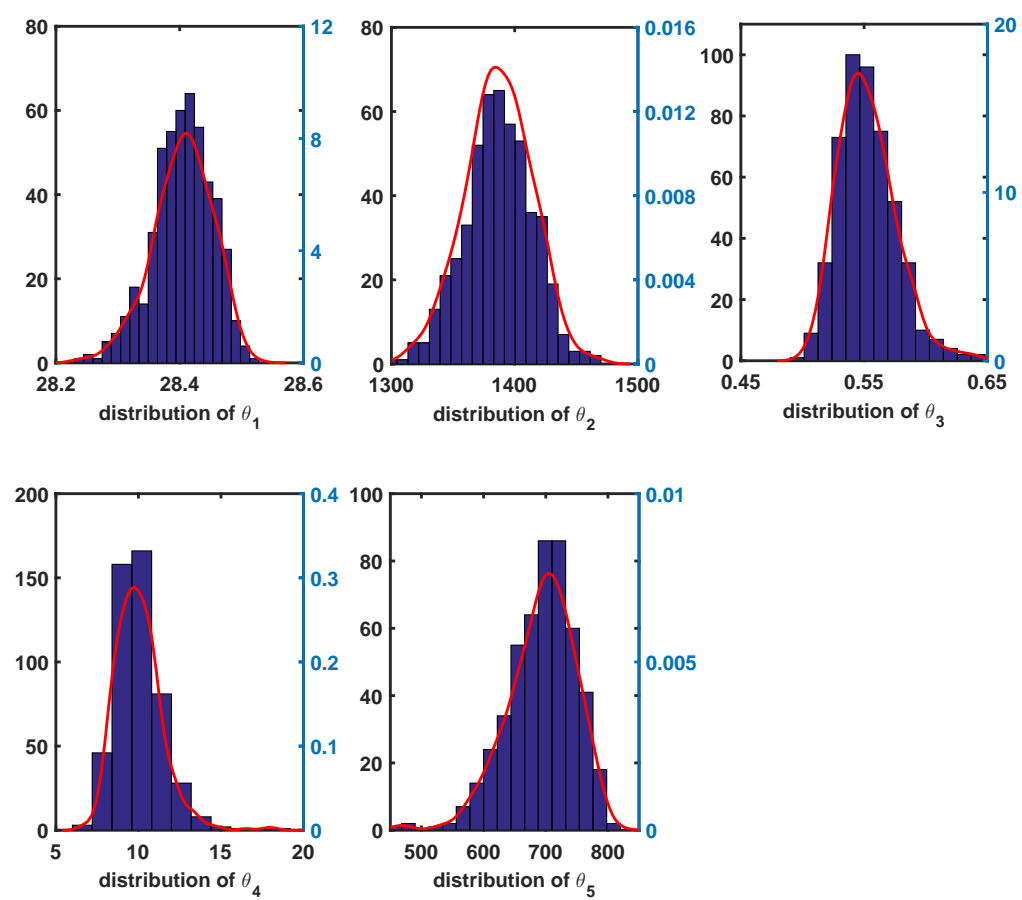

Figure 12: Histograms approximating the distribution of each parameter (left y-axis), obtained from 500 re-sampled bootstraps, using modeling approach $\mathbf{D}$ for $\mathrm{CO}_{2}$. The parameters have been fitted to $\Delta H^{v a p}$ in addition to $\rho_{\text {sat }}^{l i q}$ and $P^{s a t}$. The full red line shows the estimated probability density function (right y-axis). 


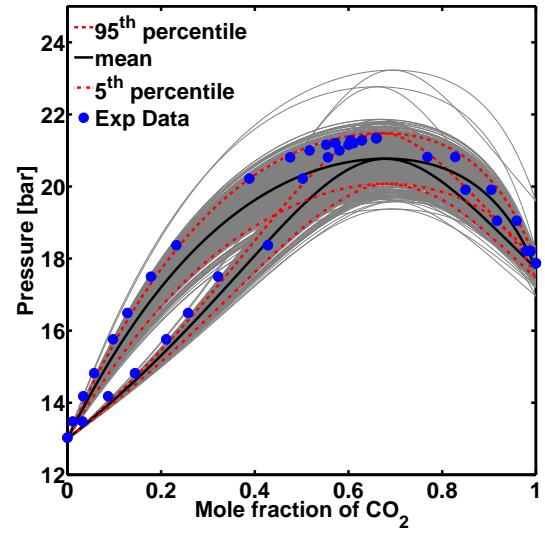

(a) Approach F.

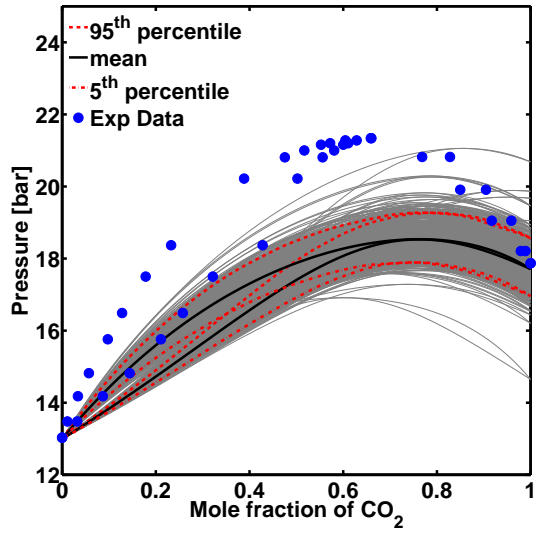

(b) Approach $\mathbf{D}$.

Figure 13: Propagated uncertainty in the model predictions for the $\mathrm{CO}_{2}+$ ethane VLE at $\mathrm{T}=250 \mathrm{~K}$. Approach $\mathbf{F}$ (a) and $\mathbf{D}$ (b) fitted to $\Delta H^{v a p}$ in addition to $\rho_{\text {sat }}^{l i q}$ and $P^{s a t}$. Grey lines represent the simulations, red dashed lines are the 5 th and 95th percentile of the simulations and black full lines are the mean of the simulations. Blue circles are experimental data from [58].

ous realizations generally under-predict the VLE data, whereas they previously over-predicted the data. This suggests a quite large total area of uncertainty, and a very flexible model. For approach $\mathrm{D}$ we see from figure $13 \mathrm{~b}$ that the uncertainty in the VLE is larger now, than it was with the bimodal distribution, however, the VLE predictions are almost identical to those in figure $9 \mathrm{~b}$. The reason for the wide parameter distributions may be because the obtained parameter sets constitute a compromise between correlating either of the fitted properties better than the other. Figure 13b, for instance, suggests that the pure compound vapour pressure is not captured very well for some parameter sets.

\section{Concluding remarks}

In this work the uncertainty in the pure compound parameters of $\mathrm{CO}_{2}$ was investigated for various modeling approaches. The models employ between three and five adjustable pure compound parameters. The uncertainties are estimated 
using either LSQ estimation or the bootstrap method. The pure compound uncertainty from the bootstrap method is propagated to selected derivative properties and $\mathrm{CO}_{2}$ +hydrocarbon VLE systems using a Monte Carlo approach.

The results indicate that modeling approaches which use only three adjustable parameters have relatively low parameter uncertainties. However, when the models contained four or five parameters the uncertainties and parameter correlations were significant. The association volume, $\beta$, for instance is highly correlated with the association strength, $\varepsilon$, and its value may vary about $100 \%$ from its mean value, without loss of accuracy in the properties the parameters are correlated to (saturated pressure and liquid density). The main reason for the large parameters uncertainties appears to be the high correlation between parameters.

It was shown that when $\mathrm{CO}_{2}$ is treated as an associating species with five parameters, the propagated uncertainty appear to be significant for highly temperature dependent properties such as the heat capacities. On the other hand the uncertainty is relatively small for the VLE systems. When qCPA was employed with four adjustable parameters, however, the uncertainties were relatively small in the temperature dependent properties, whereas the uncertainty in VLE was significant. Of the evaluated models qCPA (with approach F) is the only model which can predict the VLEs within the propagated uncertainty. That is partly due to the larger uncertainty range, but also better model predictions. This suggests that one or more parameter sets can be found, within the uncertainty of the adjustable parameters, which accurately predicts the VLE. Alternatively qCPA with three parameters gives excellent qualitative results with low uncertainties.

Although similar parameter correlations are typically obtained, the param475 eter uncertainties from LSQ estimation are generally significantly smaller than those obtained from the bootstrap method. This may be because the bootstrap method accounts for the high degree of correlation between parameters, by using the experimental data itself rather than indirectly using the linear approximation of the covariance matrix for estimators. It is thus clear that one might not 
LSQ estimation procedure. In any case, one should be aware that an obtained parameter set may be quite uncertain, which may incur significant uncertainties in physical property predictions.

The effect of adding the heat of vaporization to the parameter estimation was investigated. This resulted in parameter distributions, which were significantly closer to a normal distribution. Unfortunately the standard deviations for the parameters were still high and the propagated error is significant. qCPA with four parameters is still the only model which can, within the propagated uncertainty, predict the VLEs. The propagated uncertainty, however, differs from the previously estimated one, so that the model tend to under-predict, rather than over-predict, the VLE data.

The uncertainty results for $\mathrm{qCPA}$ are strictly valid only for $\mathrm{CO}_{2}$ and the quadrupolar term which we have employed in this work. However, most other quadrupolar and polar terms are structurally similar, and we suspect that these terms may have similar uncertainty properties. Indicating that published parameters should be treated with caution. On the other hand, it is clear from the VLE examples that the quadrupolar models may offer significantly improved predictions, even if the cost of this may be a higher uncertainty in the parameters. In either case the results indicate that it is important that researchers report the parameter uncertainties when a new model is developed or parameters are estimated for a new compound. In this way better informed decisions and comparisons can be made.

Finally we note that while we have assumed good data coverage in our analysis, it would be very informative to also analyze the effect of especially sparse or

limited data on the CPA type models as well as which types of data/properties are employed to correlate the parameters. 


\section{Appendix A. The CPA and qCPA Equations of State}

This appendix summarizes the necessary equations for the CPA and qCPA equations of state, as presented by Kontogeorgis et al. [1] and Bjørner and Kontogeorgis [33].

In terms of the reduced residual Helmholtz free energy qCPA can be expressed as:

$$
\frac{A_{C P A}^{r}(T, V, \mathbf{n})}{R T}=\frac{A_{S R K}^{r}(T, V, \mathbf{n})}{R T}+\frac{A_{A s s o c}^{r}(T, V, \mathbf{n})}{R T}+\frac{A_{Q u a d}^{r}(T, V, \mathbf{n})}{R T}
$$

where $\mathrm{T}$ is the temperature, $\mathrm{V}$ is the volume, $\mathrm{n}$ the molar composition vector and $\mathrm{R}$ the ideal gas constant.

The SRK term. The reduced residual Helmholtz energy of the SRK EoS for $\mathrm{n}$ moles of a mixture is expressed as [63]:

$$
\frac{A_{S R K}^{r}(T, V, \mathbf{n})}{R T}=-n \ln \left(1-\frac{B}{V}\right)-\frac{D(T)}{R T B} \ln \left(1+\frac{B}{V}\right)
$$

where, using the van der Waals one-fluid mixing rules

$$
\begin{aligned}
D(T) & =\sum_{i} n_{i} \sum_{j} n_{j} a_{i j}(T) \\
B & =\sum_{i} n_{i} b_{i i}
\end{aligned}
$$

bii or $b_{0}$ is the pure compound co-volume parameter for component $i$. The cross energetic parameter, aij, is calculated as

$$
a_{i j}=\sqrt{a_{i i}(T) a_{j j}(T)}\left(1-k_{i j}\right)
$$

where kij is a binary interaction parameter, typically correlated to experimental data. In this work the kij is always equal to zero. The temperature dependent energetic parameter, $a_{i i}(T)$, is given as

$$
a_{i i}(T)=a_{0}\left(1+c_{1}\left(1-\sqrt{T_{r}}\right)\right)^{2}
$$

where $\mathrm{a} 0$ and $\mathrm{c} 1$ are pure compound parameters and $\operatorname{Tr}$ is the reduced temperature $\left(=T / T_{c}\right)$. To avoid ambiguity it is common practice to tabulate the $a_{0}$ parameter as $\Gamma=a_{0} /\left(R b_{0}\right)$. 
Association contribution. The reduced residual Helmholtz energy of the association term is given by [63-65]:

$$
\frac{A_{A s s o c}^{r}(T, V, \mathbf{n})}{R T}=\sum_{i} n_{i} \sum_{A_{i}}\left(\ln X_{A_{i}}-\frac{1}{2} X_{A_{i}}+\frac{1}{2}\right)
$$

where Ai indicates bonding sites on molecule $i$ and XAi denotes the fraction of A-sites on molecule $i$ not bonded to another association site. XAi is given by

$$
X_{A_{i}}=\frac{1}{1+\frac{1}{V} \sum_{j} n_{j} \sum_{B_{j}} X_{B_{j}} \Delta^{A_{i} B_{j}}}
$$

where the association strength, DeltaAB, is calculated as

$$
\Delta^{A_{i} B_{j}}=g(n, V)\left(\exp \left(\frac{\varepsilon^{A_{i} B_{j}}}{R T}\right)-1\right) b_{i j} \beta^{A_{i} B_{j}}
$$

where eps $\mathrm{AB}$ and betaAB are the association energy and volume between site A of molecule $i$ and site $\mathrm{B}$ of molecule $j$ respectively. bij is the cross-covolume calculated from as $b_{i j}=\left(b_{i i}+b_{j j}\right) / 2$, and grad is the RDF. In CPA the RDF is approximated as

$$
g(n, V)=\frac{1}{1-1.9 \eta}
$$

where eta $(=B / 4 V)$ is the reduced fluid density.

If more than one associating compound is present the CR-1 combining rule is employed for epsAB and betaAB.

$$
\begin{aligned}
\epsilon^{A_{i} B_{j}} & =\frac{1}{2}\left(\varepsilon^{A_{i} B_{i}}+\varepsilon^{A_{j} B_{j}}\right) \\
\beta^{A_{i} B_{j}} & =\sqrt{\beta^{A_{i} B_{i}} \beta^{A_{j} B_{j}}}
\end{aligned}
$$

The association term is dependent on the association scheme (see Eq. (A.7)). To denote the different association schemes the notation of Huang and Radosz [37] is adopted.

Quadrupole contribution. The reduced residual Helmholtz energy for the quadrupolar term is based on a Padé approximation for a perturbation series

$$
\frac{A_{\text {Quad }}^{r}(T, V, \mathbf{n})}{R T}=\frac{A_{2, Q u a d}^{r}(T, V, \mathbf{n}) / R T}{1-A_{3, \text { Quad }}^{r}(T, V, \mathbf{n}) / A_{2, \text { Quad }}^{r}(T, V, \mathbf{n})}
$$


The third-order term is the sum of both a two-body and a three-body contribution, so that

$$
\frac{A_{3, \text { Quad }}^{r}(T, V, \mathbf{n})}{R T}=\frac{A_{3,2, \text { Quad }}^{r}(T, V, \mathbf{n})}{R T}+\frac{A_{3,3, \text { Quad }}^{r}(T, V, \mathbf{n})}{R T}
$$

The expressions for the second- and third-order pure compound terms developed by Larsen et al. [66] were extended to mixtures by Bjørner and Kontogeorgis [33]. The expressions are

$$
\begin{aligned}
\frac{A_{2, \text { Quad }}^{r}}{R T} & =-\frac{7}{10} \frac{N_{A}}{V\left(k_{b} T\right)^{2}} \sum_{i}^{n c} n_{i} \sum_{j}^{n c} n_{j} \frac{Q_{i j}^{4}}{\sigma_{i j}^{7}} I_{10}^{H S} \\
\frac{A_{3,2, \text { Quad }}^{r}}{R T} & =\frac{36}{245} \frac{N_{A}}{V k_{b}^{2} T^{3}} \sum_{i}^{n c} n_{i} \sum_{j}^{n c} n_{j} \frac{Q_{i j}^{6}}{\sigma_{i j}^{12}} I_{15}^{H S} \\
\frac{A_{3,3, \text { Quad }}^{r}}{R T} & =-\frac{1}{6400} \frac{N_{A}^{2}}{V^{2}\left(k_{b} T\right)^{3}} \sum_{i}^{n c} n_{i} \sum_{j}^{n c} n_{j} \sum_{k}^{n c} n_{k} \frac{Q_{i j k}^{6}}{\sigma_{i j}^{3} \sigma_{i k}^{3} \sigma_{j k}^{3}} I_{T Q}^{H S}
\end{aligned}
$$

where $N_{A}$ is the Avogadro constant, $k_{b}$ is Boltzmann's constant, $Q$ the quadrupolar moment, $\sigma$ the hard sphere diameter and $I_{n}$ and $I_{T Q}$ are correlation integrals.

It is presumed that the correlation integrals can be truncated at the zeroth order term of the density expansion in [66]. The coefficients are given as:

$$
\begin{gathered}
I_{n}^{H S} \approx J_{0, n}=\frac{4 \pi}{n-3} \\
I_{\text {tripple }}^{H S} \approx J_{0, T Q}=54 \pi^{2}
\end{gathered}
$$

For mixtures, the two- and three body cross-quadrupolar moments are calculated using from

$$
\begin{array}{r}
Q_{i j}=\sqrt{Q_{i i} Q_{j j}} \\
Q_{i j k}=\sqrt[3]{Q_{i i} Q_{j j} Q_{k k}}
\end{array}
$$

The co-volume parameter in CPA is approximated based on the molecular diameter of a hard-sphere molecule as

$$
b_{0}^{Q}=N_{A} \pi \sigma^{3} / 3
$$

where $b_{0}^{Q}$ is the co-volume parameter in the quadrupolar term. Note that the original definition of the co-volume is twice that shown in Eq. (A.19). 
Two variations of qCPA are considered in this work; one where the quadrupo-

525 i.e. $b_{0}=b_{0}^{Q}$, and one where $b_{0}^{Q}$ was used as an adjustable parameter.

\section{Appendix B. Equations for the employed derivative properties}

This appendix list expressions for the employed derivative properties; residual isochoric- and isobaric heat capacities and the residual enthalpy. The prop530

Residual isochoric heat capacity.

$$
C_{V}^{r e s}(T, V, \mathbf{n})=-R T^{2}\left(\frac{\partial^{2} F}{\partial T^{2}}\right)_{V, \mathbf{n}}-2 R T\left(\frac{\partial F}{\partial T}\right)_{V, \mathbf{n}}
$$

Residual isobaric heat capacity.

$$
C_{P}^{r e s}(T, V, \mathbf{n})=C_{V}^{r e s}-T \frac{\left(\frac{\partial P}{\partial T}\right)_{V, \mathbf{n}}^{2}}{\left(\frac{\partial P}{\partial V}\right)_{T, \mathbf{n}}} n R
$$

Residual enthalpy.

$$
\frac{H^{r e s}}{n R T}=Z-\frac{T}{n}\left(\frac{\partial F}{\partial T}\right)_{V, \mathbf{n}}-1
$$

where

$$
\begin{aligned}
& \left(\frac{\partial P}{\partial V}\right)_{T, \mathbf{n}}=-R T\left(\frac{\partial^{2} F}{\partial V^{2}}\right)_{T, \mathbf{n}}-\frac{n R T}{V^{2}} \\
& \left(\frac{\partial P}{\partial T}\right)_{V, \mathbf{n}}=-R T\left(\frac{\partial^{2} F}{\partial V \partial T}\right)_{\mathbf{n}}-\frac{P}{T}
\end{aligned}
$$

\section{Acknowledgements}

The authors gratefully acknowledge the Danish Research Council for Independent Research for funding this work as part of the project " $\mathrm{CO}_{2}$ Hydrates Challenges and Possibilities". 
[1] G. M. Kontogeorgis, E. C. Voutsas, I. V. Yakoumis, D. P. Tassios, An equation of state for associating fluids, Industrial \& Engineering Chemistry Research 35 (1996) 4310-4318. doi:10.1021/ie9600203.

URL http://pubs .acs.org/doi/abs/10.1021/ie9600203

[2] W. G. Chapman, K. E. Gubbins, G. Jackson, M. Radosz, New reference equation of state for associating liquids, In trial \& Engineering Chemistry Research 29 (8) (1990) 1709-1721. arXiv:http://dx.doi.org/10.1021/ie00104a021, doi:10.1021/ie00104a021.

URL http://dx.doi.org/10.1021/ie00104a021

[3] M. E. Reed, W. Whiting, Sensitivity and uncertainty of process design to thermodynamic model parameters: a monte carlo approach, Chemical Engineering Communications 124 (1) (1993) 39-48. arXiv:http://dx.doi.org/10.1080/00986449308936176, doi:10.1080/00986449308936176. URL http://dx.doi.org/10.1080/00986449308936176

[4] R. Dohrn, O. Pfohl, Thermophysical properties - industrial directions, Fluid Phase Equilibria 194-197 (2002) 15-29.

[5] R. S. Mah, Effects of thermophysical property estimation on process design, Computers \& Chemical Engineering 1 (3) (1977) 183 - 189. doi:http://dx.doi.org/10.1016/0098-1354(77)85003-5.

URL http://www.sciencedirect.com/science/article/pii/0098135477850035

[6] A. R. Nelson, J. H. Olson, S. I. Sandler, Sensitivity of distillation process design and operation to vle data, Industrial \& Engineering Chemistry Process Design and Development 22 (3) (1983) 547-552. arXiv:http://dx.doi.org/10.1021/i200022a034, doi:10.1021/i200022a034.

URL http://dx.doi.org/10.1021/i200022a034 
[7] A. H. Larsen, Data quality for process design, Fluid Phase Equilibria 29 (0) (1986) 47 - 58. doi:http://dx.doi.org/10.1016/0378-3812(86)85010-5. URL http://www.sciencedirect.com/science/article/pii/0378381286850105

[8] S. Zeck, Thermodynamics in process development in the chemical industry - importance, benefits, current state and future development, Fluid Phase Equilibria 70 (1991) 125-140.

[9] S. Gupta, J. D. Olson, Industrial needs in physical properties, Ind. Eng. Chem. Res 42 (2003) 6359-6374.

[10] A. Hukkerikar, S. Kalakul, B. Sarup, D. Young, G. Sin, R. Gani, Estimation of environment-related properties of chemicals for design of sustainable processes: Development of group-contribution $+(\mathrm{gc}+)$ models and uncertainty analysis, Journal of Chemical Information and Modeling 52 (11) (2012) 2823-2839, 2012 American Chemical Society. doi:10.1021/ci300350r.

[11] W. B. Whiting, T. M. Tong, M. E. Reed, Effect of uncertainties in thermodynamic data and model parameters on calculated process performance, Industrial \& Engineering Chemistry Research 32 (7) (1993) 1367-1371. arXiv:http://dx.doi.org/10.1021/ie00019a011, doi:10.1021/ie00019a011. URL http://dx.doi.org/10.1021/ie00019a011

[12] W. B. Whiting, Effects of uncertainties in thermodynamic data and models on process calculations, Journal of Chemical \& Engineering Data 41 (5) (1996) 935-941. arXiv:http://dx.doi.org/10.1021/je9600764, doi:10.1021/je9600764.

URL http://dx.doi.org/10.1021/je9600764

[13] W. B. Whiting, V. R. Vasquez, M. M. Meerschaert, Techniques for assessing the effects of uncertainties in thermodynamic models and data, Fluid Phase Equilibria, Fluid Phase Equilib 158-160 (1999) 627-641. doi:10.1016/S03783812(99)00054-0. 
[14] V. R. Vasquez, W. B. Whiting, Effect of systematic and random errors in thermodynamic models on chemical process design and simulation: A monte carlo approach, Industrial \& Engineering Chemistry Research 38 (8) (1999) 3036-3045. arXiv:http://dx.doi.org/10.1021/ie980748e, doi:10.1021/ie980748e.

URL http://dx.doi.org/10.1021/ie980748e

[15] V. R. Vasquez, W. B. Whiting, Uncertainty and sensitivity analysis of thermodynamic models using equal probability sampling (eps), Computers \& Chemical Engineering 23 (1112) (2000) 1825 - 1838. doi:http://dx.doi.org/10.1016/S0098-1354(00)00297-0.

URL http://www.sciencedirect.com/science/article/pii/S0098135400002970

[16] Y. Xin, W. B. Whiting, Case studies of computer-aided design sensitivity to thermodynamic data and models, Industrial \&amp; Engineering Chemistry Research 39 (8) (2000) 2998-3006. arXiv:http://dx.doi.org/10.1021/ie990617t, doi:10.1021/ie990617t.

URL http://dx.doi.org/10.1021/ie990617t

[17] P. M. Mathias, J. P. Gilmartin, Quantitative evaluation of the effect of uncertainty in property models on the simulated performance of solvent-based co2-capture, Energy Procedia 63 (2014) 1171 - 1185, 12th International Conference on Greenhouse Gas Control Technologies, GHGT-12. doi:http://dx.doi.org/10.1016/j.egypro.2014.11.127. URL http://www.sciencedirect.com/science/article/pii/S1876610214019420

[18] P. M. Mathias, Sensitivity of process design to phase equilibrium - a new perturbation method based upon the margules equation, Journal of Chemical \& Engineering Data 59 (4) (2014) 1006-1015. arXiv:http://dx.doi.org/10.1021/je400748p, doi:10.1021/je400748p. URL http://dx.doi.org/10.1021/je400748p

[19] P. M. Mathias, Effect of $\{$ VLE $\}$ uncertainties on the design of separation sequences by distillation - study of the benzene-chloroform- 
acetone system, Fluid Phase Equilibria 408 (2016) 265 - 272. doi:http://dx.doi.org/10.1016/j.fluid.2015.09.004.

[20] S. Hajipour, M. A. Satyro, Uncertainty analysis applied to thermodynamic models and process design - 1. pure components, Fluid Phase Equilibria 307 (1) (2011) 78 - 94. doi:http://dx.doi.org/10.1016/j.fluid.2011.05.014. URL http://www.sciencedirect.com/science/article/pii/S037838121100255X

[21] S. Hajipour, M. A. Satyro, M. W. Foley, Uncertainty analysis applied to thermodynamic models and process design - 2. binary mixtures, Fluid Phase Equilibria 364 (0) (2014) 15 - 30. doi:http://dx.doi.org/10.1016/j.fluid.2013.12.004.

URL http://www.sciencedirect.com/science/article/pii/S0378381213007097

[22] S. Hajipour, M. A. Satyro, M. W. Foley, Uncertainty analysis applied to thermodynamic models and fuel properties -natural gas dew points and gasoline reid vapor pressures, Energy \& Fuels 28 (2) (2014) 1569-1578. arXiv:http://dx.doi.org/10.1021/ef4019838, doi:10.1021/ef4019838.

URL http://dx.doi.org/10.1021/ef4019838

[23] W. A. Wakeham, G. S. Cholakov, R. P. Stateva, Consequences of property errors on the design of distillation columns, Fluid Phase Equilibria 185 (1 - 2) (2001) 1 - 12, proceedings of the 14th symposium on thermophysical properties. doi:http://dx.doi.org/10.1016/S0378-3812(01)00490-3. URL http://www.sciencedirect.com/science/article/pii/S0378381201004903

[24] J. Gross, An equation-of-state contribution for polar components: Quadrupolar molecules, AIChE Journal 51 (9) (2005) 2556-2568. doi:10.1002/aic.10502.

URL http://dx.doi.org/10.1002/aic.10502

[25] E. K. Karakatsani, I. G. Economou, Perturbed chain-statistical associating fluid theory extended to dipolar and quadrupolar molecular fluids, The Journal of Physical Chemistry B 110 (18) (2006) 
9252-9261. arXiv:http://pubs.acs.org/doi/pdf/10.1021/jp056957b, doi:10.1021/jp056957b.

URL http://pubs.acs.org/doi/abs/10.1021/jp056957b

[26] E. Karakatsani, G. Kontogeorgis, I. Economou, Evaluation of the truncated perturbed chain-polar statistical associating fluid theory for complex mixture fluid phase equilibria, Industrial \& Engineering Chemistry Research 45 (17) (2006) 6063-6074.

[27] D. NguyenHuynh, J.-P. Passarello, P. Tobaly, J.-C. de Hemptinne, Application of GC-SAFT EOS to polar systems using a segment approach, Fluid Phase Equilibria 264 (1-2) (2008) 62-75. doi:http://dx.doi.org/10.1016/j.fluid.2007.10.019.

URL http://www.sciencedirect.com/science/article/pii/S0378381207006838

[28] I. Tsivintzelis, G. M. Kontogeorgis, M. L. Michelsen, E. H. Stenby, Modeling phase equilibria for acid gas mixtures using the $\{\mathrm{CPA}\}$ equation of state. part ii: Binary mixtures with $\{\mathrm{CO} 2\}$, Fluid Phase Equilibria 306 (1) (2011) $38-56,20$ years of the $\{\mathrm{SAFT}\}$ equation of state Recent advances and challenges Symposium. doi:http://dx.doi.org/10.1016/j.fluid.2011.02.006.

URL http://www.sciencedirect.com/science/article/pii/S0378381211000641

[29] B. Efron, Bootstrap methods: Another look at the jackknife, Ann. Statist. 7 (1) (1979) 1-26. doi:10.1214/aos/1176344552.

URL http://dx.doi.org/10.1214/aos/1176344552

[30] R. Span, W. Wagner, A new equation of state for carbon dioxide covering the fluid region from the triplepoint temperature to $1100 \mathrm{k}$ at pressures up to 800 mpa, Journal of Physical and Chemical Reference Data 25 (6) (1996) 1509-1596. doi:http://dx.doi.org/10.1063/1.555991.

URL http://scitation.aip.org/content/aip/journal/jpcrd/25/6/10.1063/1.555991

[31] E. W. Lemmon, M. O. McLinden, M. L. Huber, NIST Standard Reference Database 23: Reference Fluid Thermodynamic and Transport Properties- 
REFPROP, Version 7, National Institute of Standards and Technology, Gaithersburg, standard Reference Data Program (2002).

[32] M. M. E.W. Lemmon, D. Friend, NIST Chemistry WebBook, NIST Standard Reference Database Number 69, National Institute of Standards and Technology, Gaithersburg MD, 20899, http://webbook.nist.gov, 2014, Ch. Thermophysical Properties of Fluid Systems.

URL http://webbook.nist.gov

[33] M. G. Bjørner, G. M. Kontogeorgis, Modeling derivative properties and binary mixtures with $\{\mathrm{CO} 2\}$ using the $\{\mathrm{CPA}\}$ and the quadrupolar $\{\mathrm{CPA}\}$ equations of state, Fluid Phase Equilibria 408 (2016) 151 - 169. doi:http://dx.doi.org/10.1016/j.fluid.2015.08.011.

URL http://www.sciencedirect.com/science/article/pii/S0378381215300819

[34] G. M. Kontogeorgis, M. L. Michelsen, G. K. Folas, S. Derawi, N. von Solms, E. H. Stenby, Ten years with the cpa (cubic-plus-association) equation of state. part 1. pure compounds and self-associating systems, Industrial \& Engineering Chemistry Research 45 (14) (2006) 4855-4868.

[35] G. M. Kontogeorgis, M. L. Michelsen, G. K. Folas, S. Derawi, N. von Solms, E. H. Stenby, Ten years with the cpa (cubic-plus-association) equation of state. part 2. cross-associating and multicomponent systems, Industrial \& Engineering Chemistry Research 45 (14) (2006) 4869-4878. arXiv:http://dx.doi.org/10.1021/ie051306n, doi:10.1021/ie051306n.

URL http://dx.doi.org/10.1021/ie051306n

[36] G. Kontogeorgis, G. Folas, Thermodynamic Models for Industrial Applications - From classical and advanced mixing rules to association theories, 1st Edition, John Wiley and Sons, 2010, Ch. 9-12 The Cubic Plus Association Equation of State.

[37] S. H. Huang, M. Radosz, Equation of state for small, large, polydisperse, and associating molecules, Industrial \& Engineering Chemistry Research 
29 (11) (1990) 2284-2294. arXiv:http://dx.doi.org/10.1021/ie00107a014, doi:10.1021/ie00107a014.

[38] M. Omlin, P. Reichert, A comparison of techniques for the estimation of model prediction uncertainty, Ecological Modelling 115 (1) (1999) 45 - 59.

[39] M. J. Bayarri, J. O. Berger, The interplay of bayesian and frequentist analysis, Statistical Science 19 (1) (2004) 58-80.

[40] G. A. F. Seber, C. Wild, Nonlinear Regression, John Wiley \& Sons, 1989.

[41] G. Sin, A. S. Meyer, K. V. Gernaey, Assessing reliability of cellulose hydrolysis models to support biofuel process design - identifiability and uncertainty analysis, Computers \& Chemical Engineering 34 (9) (2010) 1385 - 1392, selected papers from the 7th International Conference on the Foundations of Computer-Aided Process Design (FOCAPD), 2009, Breckenridge, Colorado, USA. doi:http://dx.doi.org/10.1016/j.compchemeng.2010.02.012.

URL http://www.sciencedirect.com/science/article/pii/S0098135410000554

[42] T. J. DiCiccio, B. Efron, Bootstrap confidence intervals, Statist. Sci. 11 (3) (1996) 189-228. doi:10.1214/ss/1032280214.

URL http://dx.doi.org/10.1214/ss/1032280214

[43] G. Sin, K. V. Gernaey, A. E. Lantz, Good modeling practice for pat applications: Propagation of input uncertainty and sensitivity analysis, Biotechnology Progress 25 (4).

725 [44] U. M. Diwekar, J. R. Kalagnanam, Efficient sampling technique for optimization under uncertainty, AIChE Journal 43 (2) (1997) 440-447. doi:10.1002/aic.690430217.

URL http://dx.doi.org/10.1002/aic.690430217 
[45] M. Mckay, R. Beckman, W. Conover, A comparison of three methods for selecting values of input variables in the analysis of output from a computer code, TECHNOMETRICS 21 (2) (1979) 239-245.

[46] R. L. Iman, W. J. Conover, A distribution-free approach to inducing rank correlation among input variables, Communications in Statistics - Simulation and Computation 11 (3) (1982) 311-334. $\quad$ arXiv:http://dx.doi.org/10.1080/03610918208812265, doi:10.1080/03610918208812265.

URL http://dx.doi.org/10.1080/03610918208812265

[47] J. C. Helton, Uncertainty and sensitivity analysis techniques for use in performance assessment for radioactive waste disposal, Reliability Engineering \& System Safety 42 (2-3) (1993) 327 - 367. doi:http://dx.doi.org/10.1016/0951-8320(93)90097-I.

URL http://www.sciencedirect.com/science/article/pii/095183209390097I

[48] N. Metropolis, S. Ulam, The monte carlo method, Journal of the American Statistical Association 44 (247) (1949) pp. 335-341.

URL http://www.jstor.org/stable/2280232

[49] J. H. Halton, A retrospective and prospective survey of the monte carlo method, SIAM Review 12 (1) (1970) pp. 1-63.

URL http://www.jstor.org/stable/2029039

[50] J. Helton, F. Davis, Latin hypercube sampling and the propagation of uncertainty in analyses of complex systems, Reliability Engineering \& System Safety 81 (1) (2003) 23 - 69. doi:http://dx.doi.org/10.1016/S09518320(03)00058-9.

URL http://www.sciencedirect.com/science/article/pii/S0951832003000589

[51] W. Duschek, R. Kleinrahm, W. Wagner, Measurement and correlation of the (pressure, density, temperature) relation of carbon dioxide ii. saturatedliquid and saturated-vapour densities and the vapour pressure along the 
entire coexistence curve, The Journal of Chemical Thermodynamics 22 (9) (1990) 841 - 864. doi:http://dx.doi.org/10.1016/0021-9614(90)90173-N.

URL http://www.sciencedirect.com/science/article/pii/002196149090173N

[52] E. Fernádez-Fassnacht, F. D. Río, The vapour pressure of $\mathrm{co}_{2}$ from 194 to $243 \mathrm{k}$, The Journal of Chemical Thermodynamics 16 (5) (1984) 469 - 474. doi:http://dx.doi.org/10.1016/0021-9614(84)90204-0.

URL http://www.sciencedirect.com/science/article/pii/0021961484902040

[53] J. R. Roebuck, T. A. Murrell, E. E. Miller, The joule-thomson effect in carbon dioxide, Journal of the American Chemical Society 64 (2) (1942) 400-411. arXiv:http://dx.doi.org/10.1021/ja01254a048, doi:10.1021/ja01254a048.

URL http://dx.doi.org/10.1021/ja01254a048

[54] S. G. Sauer, W. G. Chapman, A parametric study of dipolar chain theory with applications to ketone mixtures, Industrial \& Engineering Chemistry Research 42 (22) (2003) 5687-5696. arXiv:http://dx.doi.org/10.1021/ie034035u, doi:10.1021/ie034035u.

URL http://dx.doi.org/10.1021/ie034035u

[55] S. Korden, N. Van Nhu, J. Vrabec, J. Gross, K. Leonhard, On the treatment of electrostatic interactions of non-spherical molecules in equation of state models, Soft Materials 10 (1-3) (2012) 81-105. $\quad$ arXiv:http://dx.doi.org/10.1080/1539445X.2011.599701, doi:10.1080/1539445X.2011.599701.

URL http://dx.doi.org/10.1080/1539445X.2011.599701

[56] A. de Villiers, C. Schwarz, A. Burger, G. Kontogeorgis, Evaluation of the PC-SAFT, SAFT and CPA equations of state in predicting derivative properties of selected non-polar and hydrogenbonding compounds, Fluid Phase Equilibria 338 (0) (2013) 1-15. doi:http://dx.doi.org/10.1016/j.fluid.2012.09.035.

URL http://www.sciencedirect.com/science/article/pii/S0378381212004839 
[57] U. K. Deiters, K. M. D. Reuck, Guidelines for publication of equations of state i. pure fluids: Technical report, Chemical Engineering Journal 69 (1) (1998) 69 - 81. doi:http://dx.doi.org/10.1016/S1385-8947(97)00070-3.

URL http://www.sciencedirect.com/science/article/pii/S1385894797000703

[58] M. S.-W. Wei, T. S. Brown, A. J. Kidnay, E. D. Sloan, Vapor + liquid equilibria for the ternary system methane + ethane + carbon dioxide at $230 \mathrm{k}$ and its constituent binaries at temperatures from 207 to 270 k, Journal of Chemical \&amp; Engineering Data 40 (4) (1995) 726-731. arXiv:http://dx.doi.org/10.1021/je00020a002, doi:10.1021/je00020a002.

URL http://dx.doi.org/10.1021/je00020a002

[59] L. A. Webster, A. J. Kidnay, Vapor-liquid equilibria for the methan-propane-carbon dioxide systems at $230 \mathrm{k}$ and $270 \mathrm{k}$, Journal of Chemical \&amp; Engineering Data 46 (3) (2001) 759-764. arXiv:http://dx.doi.org/10.1021/je000307d, doi:10.1021/je000307d.

URL http://dx.doi.org/10.1021/je000307d

[60] T. Lafitte, D. Bessieres, M. M. Pieiro, J.-L. Daridon, Simultaneous estimation of phase behavior and second-derivative properties using the statistical associating fluid theory with variable range approach, The Journal of Chemical Physics 124 (2) (2006) -. doi:http://dx.doi.org/10.1063/1.2140276.

URL http://scitation.aip.org/content/aip/journal/jcp/124/2/10.1063/1.2140276

[61] G. M. Kontogeorgis, I. Tsivintzelis, N. von Solms, A. Grenner, D. Bøgh, M. Frost, A. Knage-Rasmussen, I. G. Economou, Use of monomer fraction data in the parametrization of association theories, Fluid Phase Equilibria 296 (2) (2010) 219 - 229, \{VIII\} Ibero-American Conference on Phase Equilibria and Fluid Properties for Process Design. doi:http://dx.doi.org/10.1016/j.fluid.2010.05.028.

URL http://www.sciencedirect.com/science/article/pii/S0378381210002840

[62] P. C. Tybjerg, G. M. Kontogeorgis, M. L. Michelsen, E. H. Stenby, Phase 
equilibria modeling of methanol-containing systems with the $\{\mathrm{CPA}\}$ and spc-saft equations of state, Fluid Phase Equilibria 288 (12) (2010) 128 138. doi:http://dx.doi.org/10.1016/j.fluid.2009.10.021.

URL http://www.sciencedirect.com/science/article/pii/S0378381209004312

[63] M. L. Michelsen, J. M. Mollerup, Thermodynamic Models: Fundamentals \& Computational aspects, 2nd Edition, Tie-Line Publications, 2007.

[64] G. Jackson, W. G. Chapman, K. E. Gubbins, Phase equilibria of associating fluids, Molecular Physics 65 (1) (1988) 1-31.

[65] W. G. Chapman, G. Jackson, K. E. Gubbins, Phase equilibria of associating fluids, Molecular Physics 65 (5) (1988) 1057-1079.

[66] B. Larsen, J. Rasaiah, G. Stell, Thermodynamic perturbation theory for multipolar and ionic liquids, Molecular Physics 33 (4) (1977) 987-1027. arXiv:http://www.tandfonline.com/doi/pdf/10.1080/00268977700100901, doi:10.1080/00268977700100901.

URL http://www . tandfonline.com/doi/abs/10.1080/00268977700100901 